TAO, Vol. 16, No. 5, 1133-1161, December 2005

\title{
Dynamics of Orographic Rain Associated with the Passage of a Tropical Cyclone over a Mesoscale Mountain
}

\author{
Nicholas C. Witcraft ${ }^{1}$, Yuh-Lang Lin ${ }^{1, *}$, and Ying-Hwa Kuo ${ }^{2}$ \\ (Manuscript received 12 October 2004, in final form 15 September 2005)
}

\begin{abstract}
In this study, the Penn State/NCAR Mesoscale Model Version 5 (MM5) was used to simulate Supertyphoon Bilis (2000) in order to improve the prediction and the understanding of dynamics of orographic rainfall associated with the passage of typhoons over the Central Mountain Range (CMR) of Taiwan. The rainfall prediction is significantly improved by bogussing a vortex, compared to a previous study (Lin et al. 2002). We identified several common ingredients present in this case which are responsible for producing heavy rainfall. The most important ingredients appear to be the presence of convective available potential energy (CAPE), a potentially unstable layer, a very moist airstream impinging on the CMR, and the presence of a low level wind maximum associated with the outer circulation of the typhoon. A simple moisture flux model was also used to estimate rainfall, which compares well with observations. In addition, we also found that the orographic rainfall is dominated by moisture convergence and the contribution of moisture advection in the vicinity of the mountain is negligible.
\end{abstract}

(Key words: Supertyphoon Bilis, Typhoon, Rainfall prediction, CAPE, CMR, Orographic rainfall, MM5)

\section{INTRODUCTION}

Taiwan is extremely vulnerable to the influences of tropical cyclones (TCs). In a typical year, three to four typhoons have an impact on the island nation, with one or two making

\footnotetext{
${ }^{1}$ Department of Marine, Earth, and Atmospheric Sciences, North Carolina State University, Raleigh, North Carolina, USA

2 Division of Microscale and Mesoscale Meteorology, National Center for Atmospheric Research, Boulder, Colorado, USA

* Corresponding author address.Dr. Yuh-Lang Lin, Department of Marine, Earth, and Atmospheric Sciences, North Carolina State University, Raleigh, North Carolina, USA; E-mail: yl_lin@ncsu.edu
} 
landfall. However, a typhoon does not need to make direct landfall for Taiwan to experience damaging winds and heavy rains. The forecasting of typhoons around Taiwan is complicated by the rugged topography of Taiwan's Central Mountain Range (CMR), which has an average elevation of $3000 \mathrm{~m}$ and a dimension of $300 \mathrm{~km} \times 100 \mathrm{~km}$. The isolated nature of Taiwan makes it an ideal environment for research into the orographic effects on tropical cyclones. Lin (1993) and Wu and Kuo (1999) provide thorough reviews of previous studies and current understanding of typhoons that effect Taiwan. The challenge of predicting heavy rainfall associated with TCs affecting Taiwan is the primary motivation of this study.

A TC affecting Taiwan satisfies many of common ingredients for heavy orographic rainfall proposed by Lin et al. (2001). These ingredients are: 1) steep mountain orography, 2) a low level jet, 3) high moisture upstream, 4) a high precipitation efficiency of the incoming airstream, 5) a conditionally or potentially unstable airstream upstream, 6) favorable mountain geometry (i.e., a concave region) to assist in the convergence of the flow, 7) strong synoptically forced upward vertical motion, 8) a large convective system, 9) slow movement of the convective system. We will use fine scale simulations of Supertyphoon Bilis (2000) to demonstrate that most of these ingredients were satisfied for that case. Based on the above common ingredients, Lin et al. (2002) proposed the use of a flux model to estimate orographic rainfall and distribution. One of these is the orographically induced moisture flux, $(\boldsymbol{V} \cdot \nabla h) q$, where $\boldsymbol{V}$ is the flow impinging on the mountain, $h$ the terrain height, and $q$ the water vapor mixing ratio of the incoming flow (Lin et al. 2001). This flux, along with the general vertical moisture flux, $w q$, will be calculated and compared with both observed and model derived rainfall. This will also help us identify the most essential ingredients among the nine common ingredients for orographic rainfall associated with the passage of a typhoon over CMR. In addition, we will derive and use a more rigorous form of the moisture flux, in which the convergence and advection terms are separated.

Great strides have been made in the numerical modeling of tropical cyclones (TCs) in the last decade. Major improvements in simulating TCs have been seen by reducing the grid spacing below $10 \mathrm{~km}$, while grid spacing less than $5 \mathrm{~km}$ is able to resolve fine-scale structure such as the eye wall, feeder bands, and storm outflow (Tripoli 1992; Liu et al. 1997; Braun and Tau 2000; Davis and Bosart 2002). By using higher resolution one can avoid the use of convective parameterization. This is advantageous because the track of TCs has been shown to be highly sensitive to cumulus parameterization (Davis and Bosart 2002). With this in mind, we plan to improve rainfall prediction by reducing grid resolution to $2.33 \mathrm{~km}$ from the $5 \mathrm{~km}$ resolution used in Lin et al. (2002).

There is a growing body of research concerning the simulation of TCs affecting Taiwan. Early modeling efforts were mostly idealized simulations (Chang 1982; Yeh and Elsberry 1993b; Smith and Smith 1995; Lin et al. 1997, 2001). More recently, mesoscale models have been used to simulate real TCs. For example, Wu (2001) used the GFDL model to produce an accurate track for Typhoon Gladys (1994). Rainfall amounts were also well simulated, though the distribution and location were somewhat different from observations. Wu et al. (2002) used the Penn State/NCAR mesoscale model MM5 to produce an accurate simulation of Typhoon Herb (1996). Modeled rainfall improved as the resolution was increased. The $2.2 \mathrm{~km}$ domain rainfall was superior, but was still $30 \%$ less than the observed rainfall. Lin et al. (2001) 
simulated Bilis (2000) by using the US Navy COAMPS model with Rutledge and Hobbs microphysical parameterization scheme. The modeled track compares well with the observed track; however, the modeled rainfall in that study was much greater than what was observed. A common understanding among the forecasters and scientists in Taiwan is that as long as the track is well-predicted, the prediction of rainfall, in terms of amounts and distribution, is less a problem. However, due to the extremely high terrain, accurate prediction of rainfall induced by terrain during the passage of a typhoon remains challenging. One objective of this study is to improve the prediction of rainfall by bogusing a vortex initially, which was not practiced by Lin et al. (2001).

This paper is organized as follows. Section 2 will discuss the synoptic environments and the MM5 model configuration. In section 3, we will discuss control simulation, compare the predicted rainfall with observations, identify the most essential ingredients and discuss the relative importance of moisture convergence and moisture advection on orographic rainfall production. Concluding remarks will be made in section 4 .

\section{SUMMARY OF SUPERTYPHOON BILIS, MODEL DESCRIPTION, AND EX- PERIMENT DESIGN}

\subsection{Summary of the Observed Tropical Cyclone}

Supertyphoon Bilis (2000) formed as a tropical depression northwest of Yap Island at 1200 UTC 18 August 2000. It followed an almost straight northwesterly track approaching Taiwan and became a very intense Category 5 typhoon with a minimum pressure of $915 \mathrm{mb}$ as it made landfall on the southeast coast of Taiwan near Taitung $\left(121.4^{\circ} \mathrm{E}, 23.1^{\circ} \mathrm{N}\right)$ around $1400 \mathrm{UTC}$ 22 August 2000. The best track from the Joint Typhoon Warning Center is shown in Fig. 1. The storm produced a maximum wind of $70 \mathrm{~m} \mathrm{~s}^{-1}$ and heavy rainfall of $949 \mathrm{~mm}$ in $20 \mathrm{hrs}$ measured at $\mathrm{Yu}-\mathrm{Li}$ in northeast Taiwan. Several people died or were seriously hurt by mudslides triggered by the heavy rainfall. Just before landfall, Bilis turned north and followed a cyclonic track across the island (Witcraft 2004), which is similar to many previous observed and simulated TCs passing over Taiwan (Wang 1980; reviewed briefly in Lin et al. 1999). A welldefined eye was noted with Bilis as it approached Taiwan (Fig. 2a). After landfall in Taiwan, it weakened rapidly and made landfall on the southeast coast of China around 1200 UTC 23 August. The interaction with the high terrain of the CMR helped to significantly weaken Bilis. Satellite imagery (Fig. 2b) illustrates this beautifully.

Figure 3a shows the observed synoptic environment across eastern Asia and the northwestern Pacific Ocean at 0000 UTC 22 August 2000 (denoted as 22/00Z) from Taiwan's Central Weather Bureau (CWB). Another tropical system, Tropical Storm Kaemi, was near the central coast of Vietnam, and made landfall 12 hours later and dissipated inland on the Indo-China Peninsula. To the north and northeast of Bilis there was a persistent but spatially small area of high pressure that moved generally westward through the region of the outer coarse model domain into the middle domain by the end of the simulation period. These weather systems, along with Tropical Storm Kaemi and the north Pacific high, tended to help advect or 


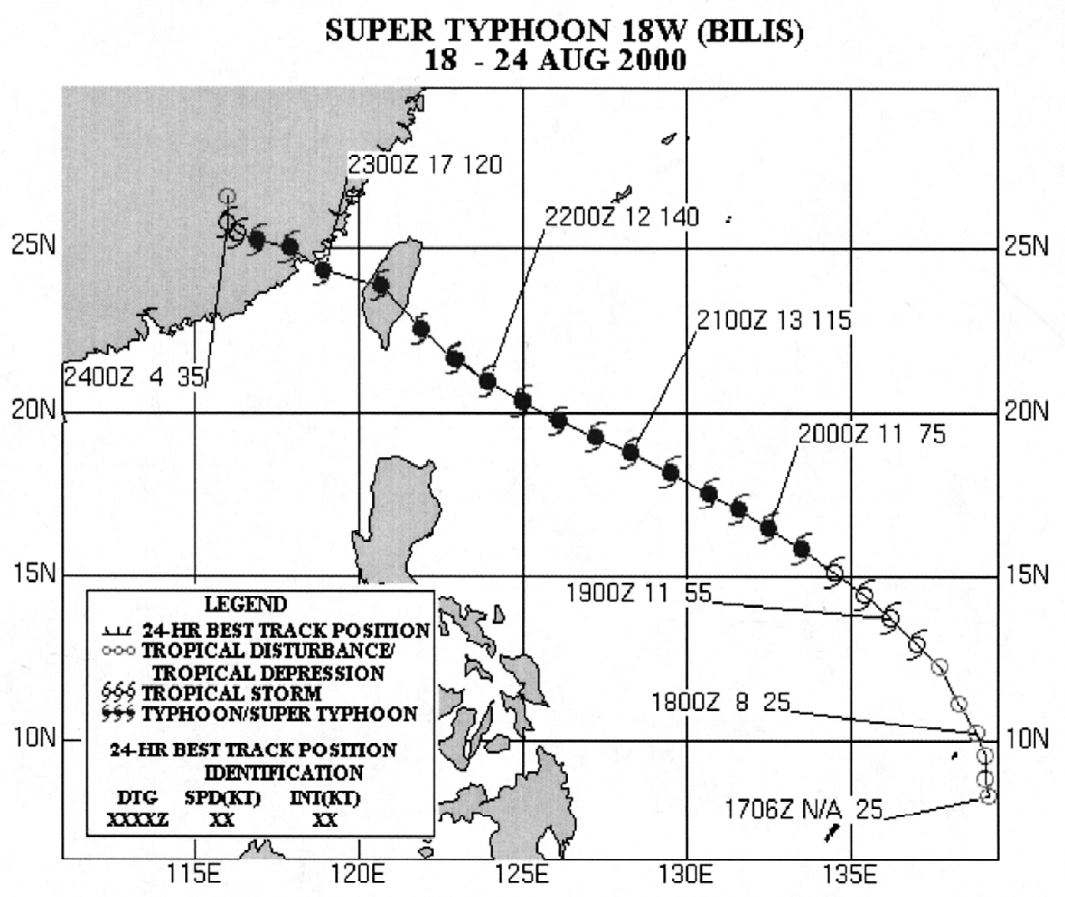

Fig. 1. 6-hourly positions of Supertyphoon Bilis (from the Joint Typhoon Warning Center).

steer Bilis northwestward toward Taiwan. There were no mid-latitude frontal features in the area to influence the track and rainfall of Bilis. Similar features can be seen in the $700 \mathrm{mb}$ analysis (Fig. 3b). On the $700 \mathrm{mb}$ analysis a low level jet (LLJ) can be seen associated with the circulation of Bilis, impinging on the CMR. It will be shown later that the LLJ was a significant factor in the formation of heavy rainfall over Taiwan. The $500 \mathrm{mb}$ and $300 \mathrm{mb}$ charts (Figs. 3c, d) show an upper-level high pressure centered halfway between Taiwan and Japan, with general ridging extending to the east and west. This persistent feature helped to steer Bilis to the northwest toward Taiwan, and also to shield Bilis from the trough to the north.

\subsection{Model Description and Experiment Design}

To simulate Supertyphoon Bilis, we used the Penn State/NCAR MM5 v3.5 model. The model solves the fully compressible, nonhydrostatic governing equations in the $\sigma$ - z vertical coordinates. Details of the model can be found in Duhdia (1996) and Grell et al. (1994). The Blackadar scheme is used to parameterize the planetary boundary layer (PBL) processes including surface fluxes and friction (Zhang and Anthes 1982). The 1-km resolution terrain and land use data used in this study provides a much higher terrain resolution than that used in most previous studies. 

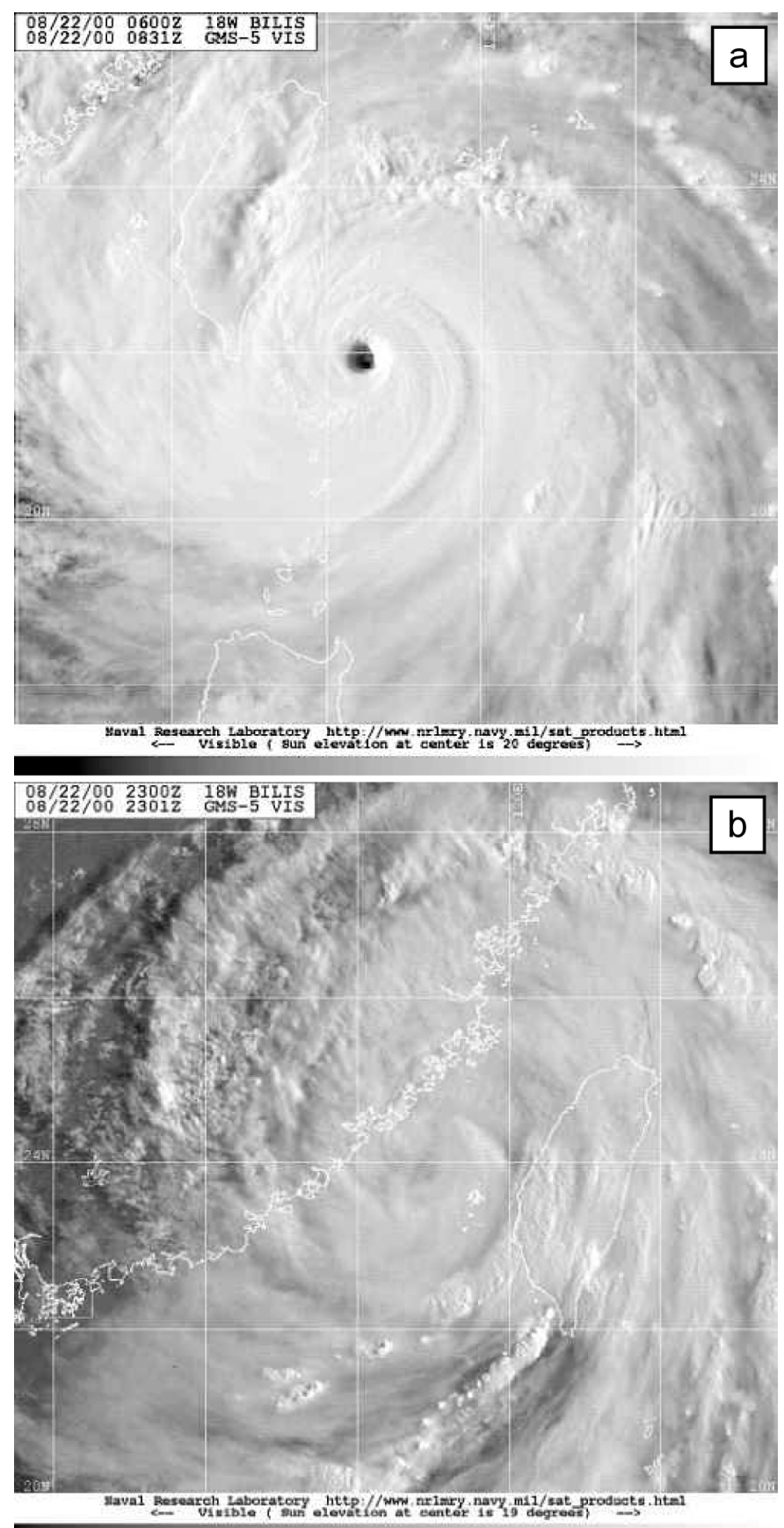

Fig. 2. Visible satellite images of Bilis for (a) before landfall (0830 UTC 22 August 2000) and (b) after landfall (2300 UTC 22 August 2000). 

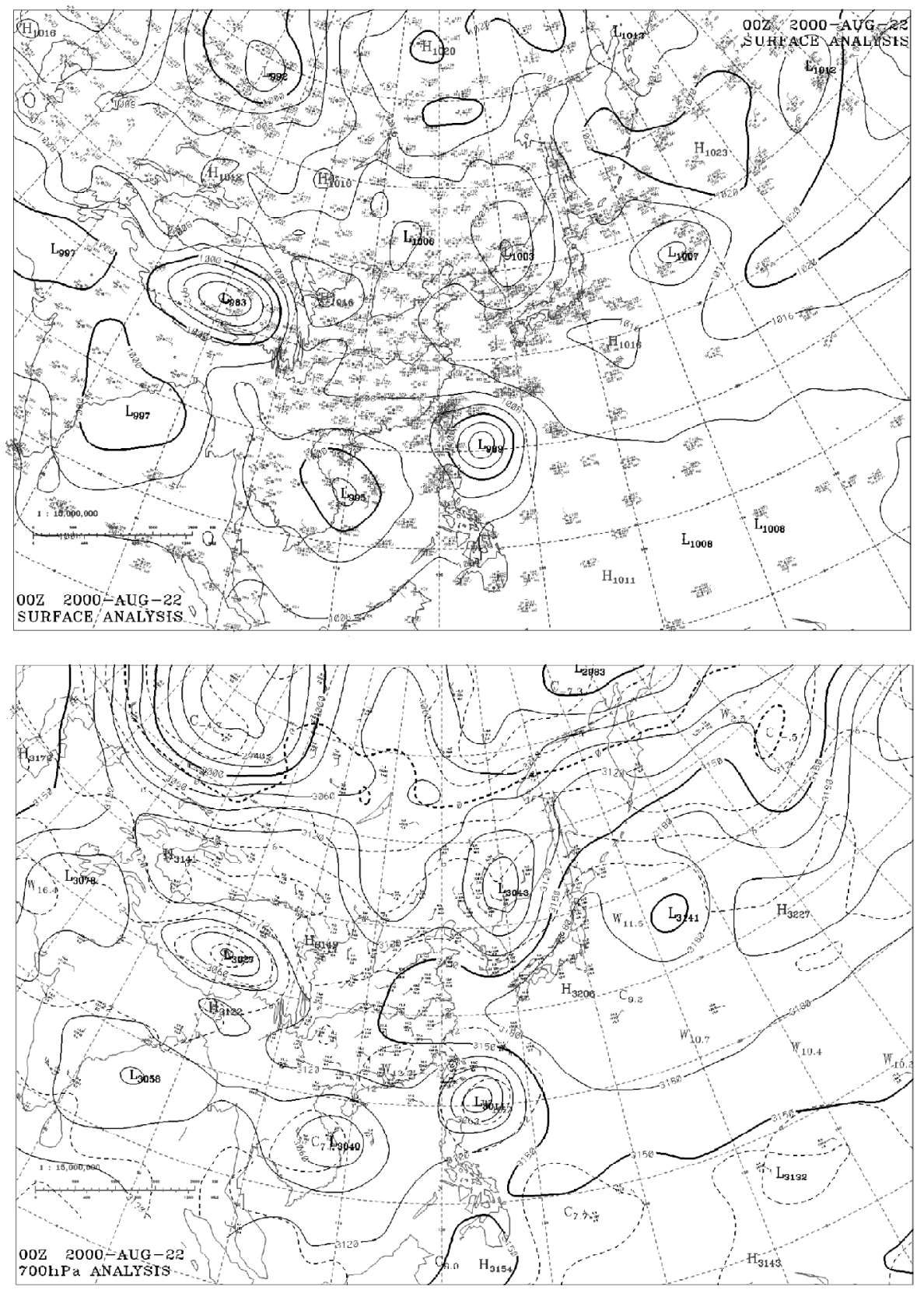

Fig. 3. Analysis fields for 0000 UTC 22 August 2000: (a) surface analysis, (b) $700 \mathrm{mb}$ analysis, (c) $500 \mathrm{mb}$ geopotential height field, and (d) $300 \mathrm{mb}$ geopotential height field. 

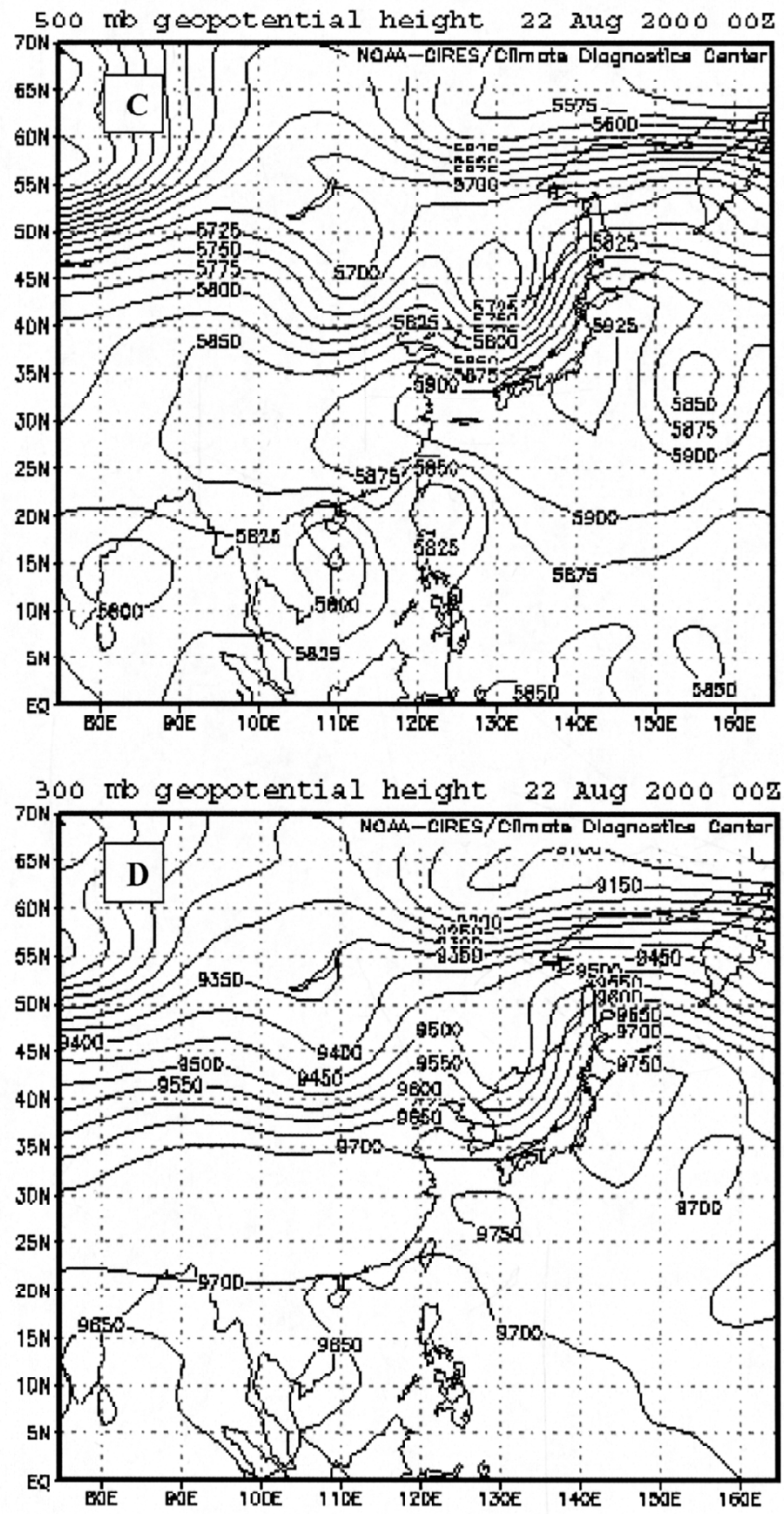

Fig. 3. Continued. 
The National Center for Environmental Prediction (NCEP) operational analysis with a resolution of $2.5^{\circ} \times 2.5^{\circ}$ latitude-longitude and 15 standard pressure layers is used for model initialization and for boundary conditions during model integration. The sea surface temperatures were initialized from the navy SST (NAVSST) analysis. All experiments are integrated for a period of 48 hours from 1200 UTC 21 August to 1200 UTC 23 August 2000. Because the initial data is too coarse to properly capture a tropical cyclone, the re-analysis vortex is removed and a bogus vortex with $70 \mathrm{~m} \mathrm{~s}^{-1}$ maximum tangential wind is inserted using the bogussing scheme of Low-Nam and Davis (2001).

There are three nested domains designed for the simulations (Fig. 4a). Domain 1 uses a $21-\mathrm{km}$ grid spacing and $200 \times 200$ grid points in the horizontal. Domain 2 uses a 7-km grid spacing and $199 \times 199$ grid points. Domain 3 uses a $2.33-\mathrm{km}$ grid spacing and $265 \times 244$ grid points. The terrain for domain 3 is shown in Fig. 4b. There are 33 sigma levels with high resolution in the boundary layer. The top of the model is at $50 \mathrm{mb}$. The time steps for domains 1, 2, and 3 are 30, 10, and 4 s, respectively. Goddard-Lin scheme (Lin et al. 1983; Tao and Simpson 1993) is used to parameterize grid-scale microphysical processes in all domains. The Betts-Miller (1986) scheme is used to parameterize subgrid-scale convection at $21 \mathrm{~km}$, while no cumulus scheme was used at 7 and $2.33 \mathrm{~km}$. The $21 \mathrm{~km}, 7 \mathrm{~km}$, and $2.33 \mathrm{~km}$ simulations are referred to as CON21, CON7, and CON2 in the rest of the paper, respectively.

\section{OROGRAPHIC RAIN FORMATION AND DISTRIBUTION}

\subsection{Comparison of the 3 Simulations}

To obtain accurate rainfall prediction for a tropical system passing over complex terrain, one needs a cyclone track that is very close to observations. For Typhoon Bilis, a control run has been performed where the model track closely matches the observed track in the $21 \mathrm{~km}, 7 \mathrm{~km}$, and $2.33 \mathrm{~km}$ domains. Figure 5 shows the observed track and model tracks CON21, CON7, and CON2, representing the $21 \mathrm{~km}, 7 \mathrm{~km}$, and $2.33 \mathrm{~km}$ domains, respectively. The timing of model landfalls lags the observed landfall by less than 1 hour. The farthest point of landfall was approximately $40 \mathrm{~km}$ south of observations for CON21, while those of CON7 and CON2 were even closer. Given the small track error in all domains, a meaningful rainfall distribution should be obtained for all domains.

Figure 6 shows the observed 24-hour rainfall ending at 0000 UTC 23 August 2000. An extensive area of rainfall in excess of $200 \mathrm{~mm}$ is present on the east slopes of the CMR to the north of Taitung, where Bilis made landfall. Of the areas north of Taitung, there were two areas of maximum rainfall. The northern maximum was near $700 \mathrm{~mm}$, and was associated with outer parts of the typhoon circulation impinging on this portion of the CMR. A radar image at 1200 UTC 22 August (Fig. 7) shows a prominent feeder band impinging on this general area. A southern maximum of nearly $500 \mathrm{~mm}$ is located just north of the landfall area, where the intense eyewall convection would have made landfall. Another maximum of approximately $300 \mathrm{~mm}$ is located to the south of where the center crossed Taiwan. This area would have experienced upslope westerly flow on the south side of the circulation as the 

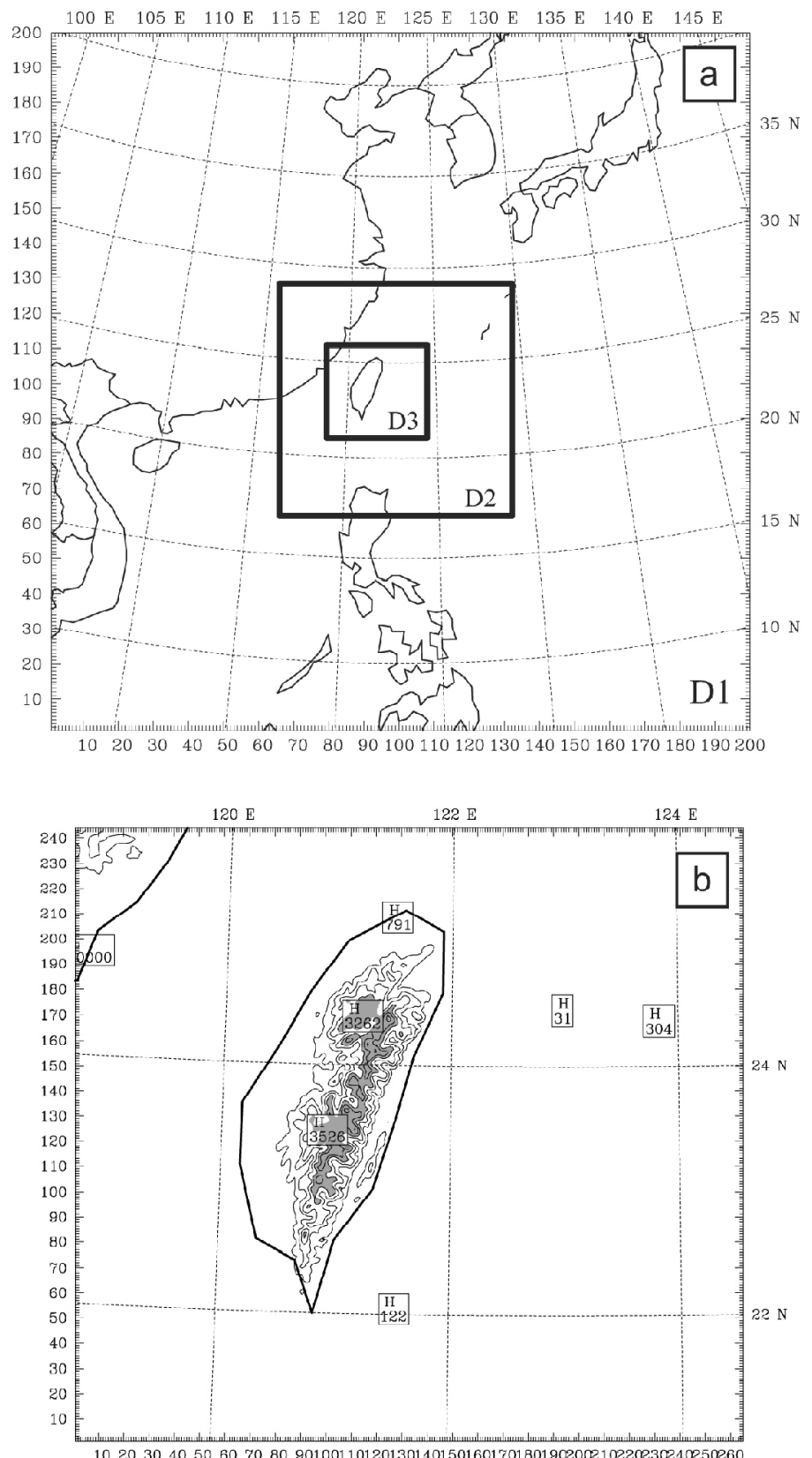

Fig. 4. (a) Domain configuration. Domain 1 (D1) has $21-\mathrm{km}$ grid spacing, $200 \times 200$, domain 2 (D2) has 7-km grid spacing, $199 \times 199$, and domain 3 (D3) has 2.3-km grid spacing, $265 \times 244$. (b) Terrain (contour interval $500 \mathrm{~m}$ ) used for the $2.3-\mathrm{km}$ grid spacing domain. Shaded areas denote elevations exceeding $2000 \mathrm{~m}$. 


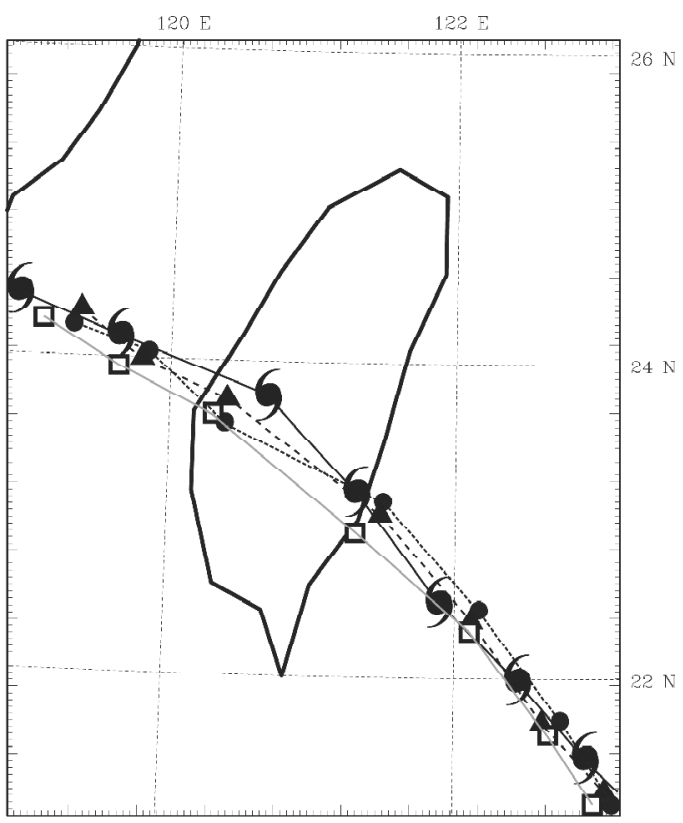

Fig. 5. Comparison of the 3 hourly low center tracks to observations. Observed track is denoted by the hurricane symbol, CON21 by squares, CON7 by triangles, and CON2 by bullets.

typhoon crossed over Taiwan.

The rainfall simulated in CON21 was generally too low. Figure 8 shows the total 24-hour rainfall, produced by the Betts-Miller cumulus parameterization scheme plus the Goddard-Lin grid-explicit microphysical parameterization scheme. Maximum rainfall amounts to the north of Taitung are slightly more than half of observations. The maximum rainfall in the south is close to observations. The general distribution of rainfall over $200 \mathrm{~mm}$ is also well predicted. The under-prediction of rain in the northern CMR is likely partly due to resolution.

Reducing the resolution to $7 \mathrm{~km}$ has the effect of increasing the rainfall amounts. Maximum 24-hour rainfall ending 30 Aug 0000 UTC for CON7 (Fig. 9) was $670 \mathrm{~mm}$ in the central CMR, with amounts exceeding $500 \mathrm{~mm}$ in the highest terrain in both the northern CMR and the southern CMR. The maximum of $670 \mathrm{~mm}$ was not in the correct location, but was close to the maximum 24-hour rainfall observed for all of Taiwan. Amounts in the southern CMR were grossly over-predicted, by nearly a factor of 2 . The southern maximum is consistently overpredicted no matter what model configuration is used.

CON2 produced highly detailed rainfall distribution. At 2.33-km resolution, one can see the effect of individual mountains on producing local rainfall maxima. The distribution of 24hour rainfall (Fig. 10) is quite similar to CON7. The area of rainfall over $200 \mathrm{~mm}$ is similar. Rainfall amounts over the ocean are very similar. The major differences of CON2 versus CON21 and CON7 were in the maximum amounts over the mountains, due to the lower grid spacing and higher model terrain. The maximum rainfall was $844 \mathrm{~mm}$, and was located very 


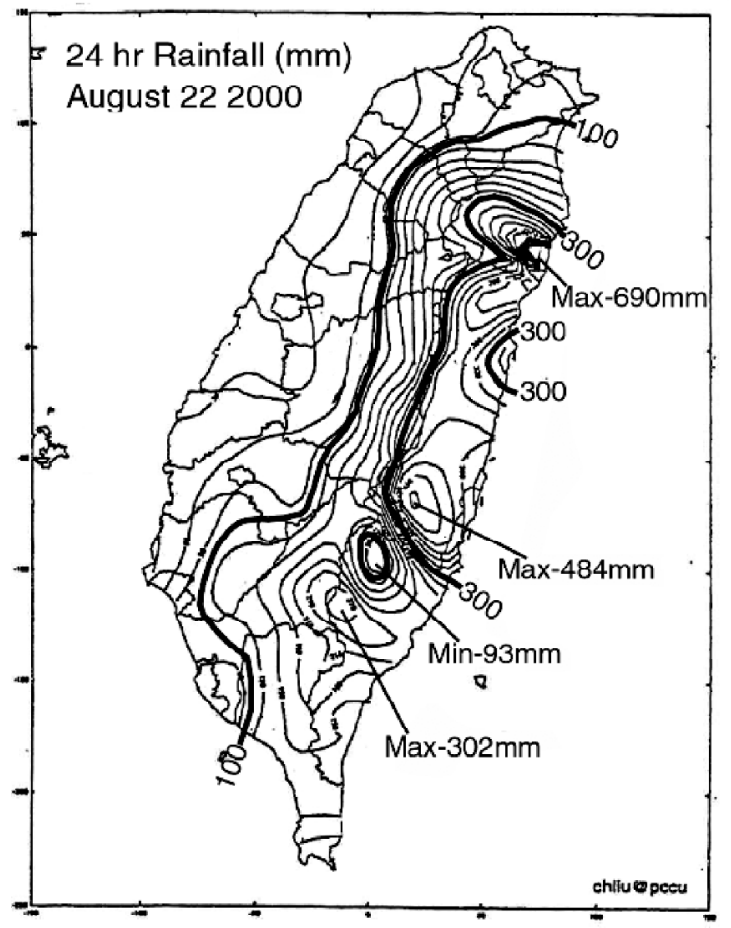

Fig. 6. Observed 24-hour rainfall over Taiwan on 22 August 2000.

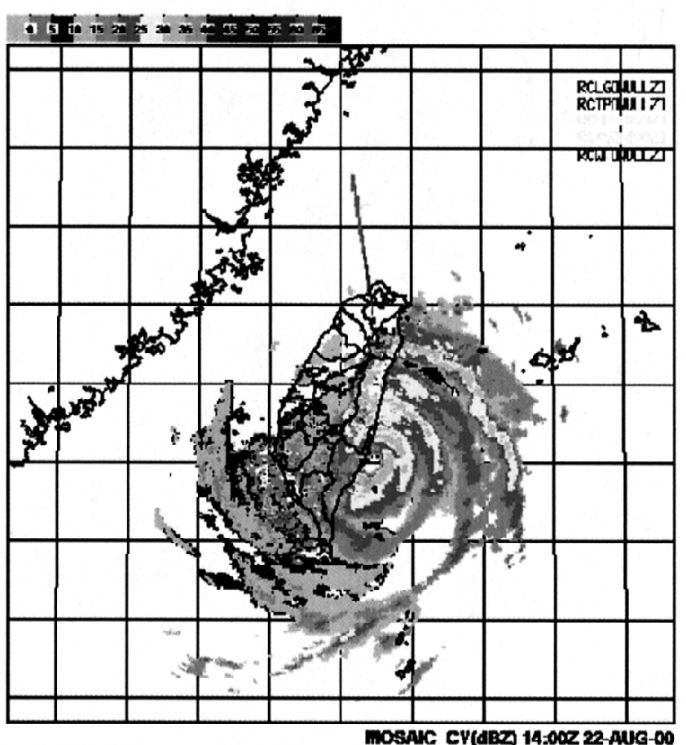

Fig. 7. Radar image of Bilis at landfall 1400 UTC 22 August 2000. 


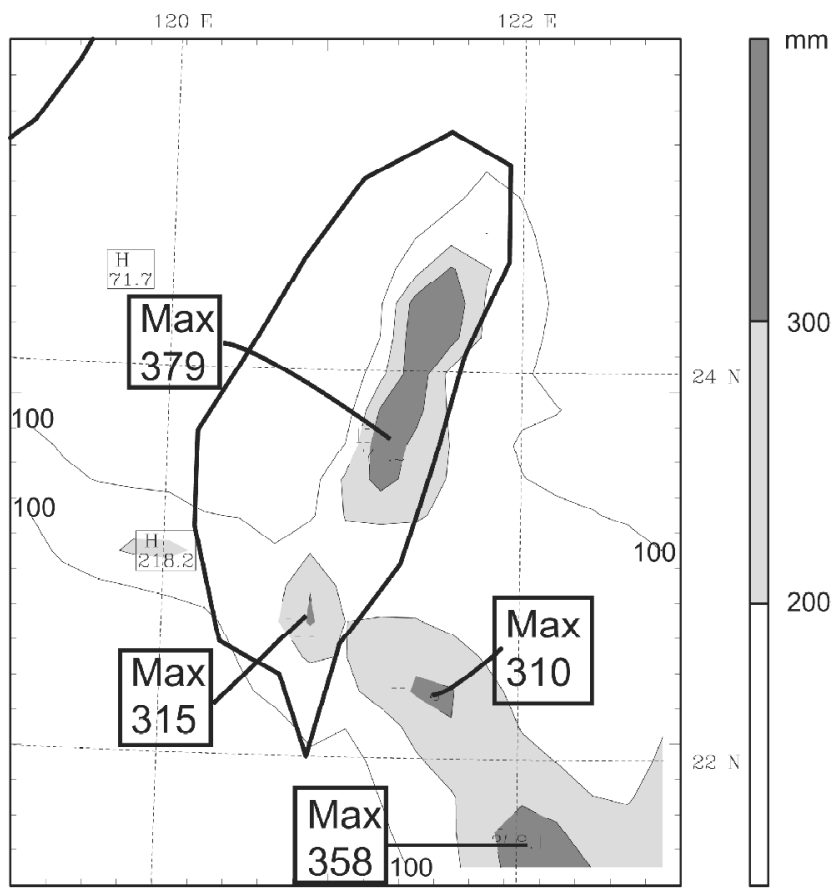

Fig. 8. 24-hour rainfall ending 0000 UTC 23 August 2000 for case CON21.

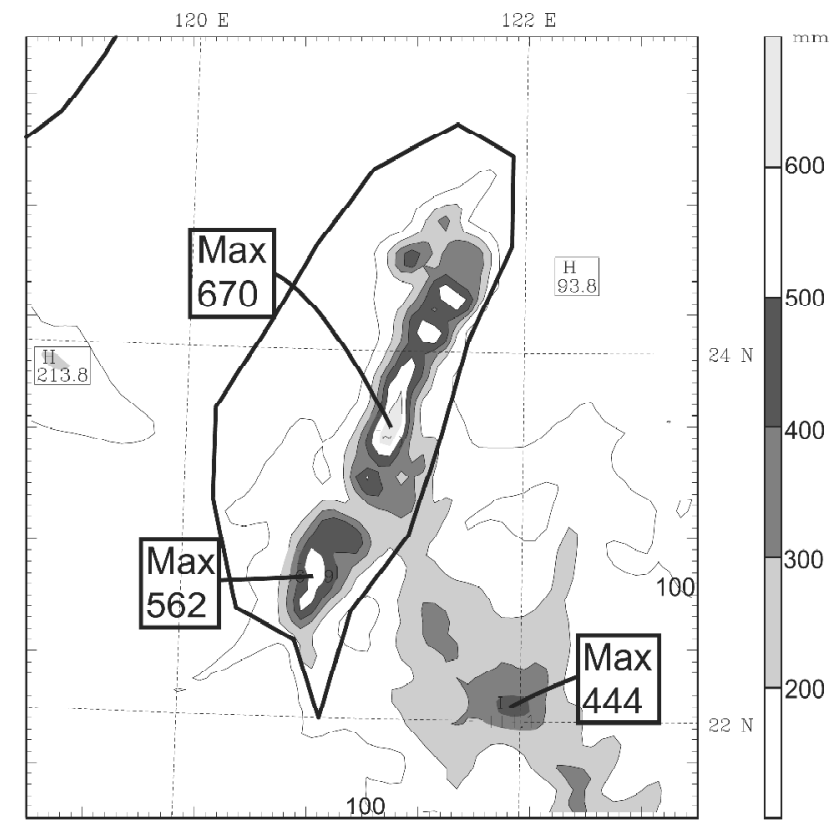

Fig. 9. 24-hour rainfall ending 0000 UTC 23 August 2000 for case CON7. 


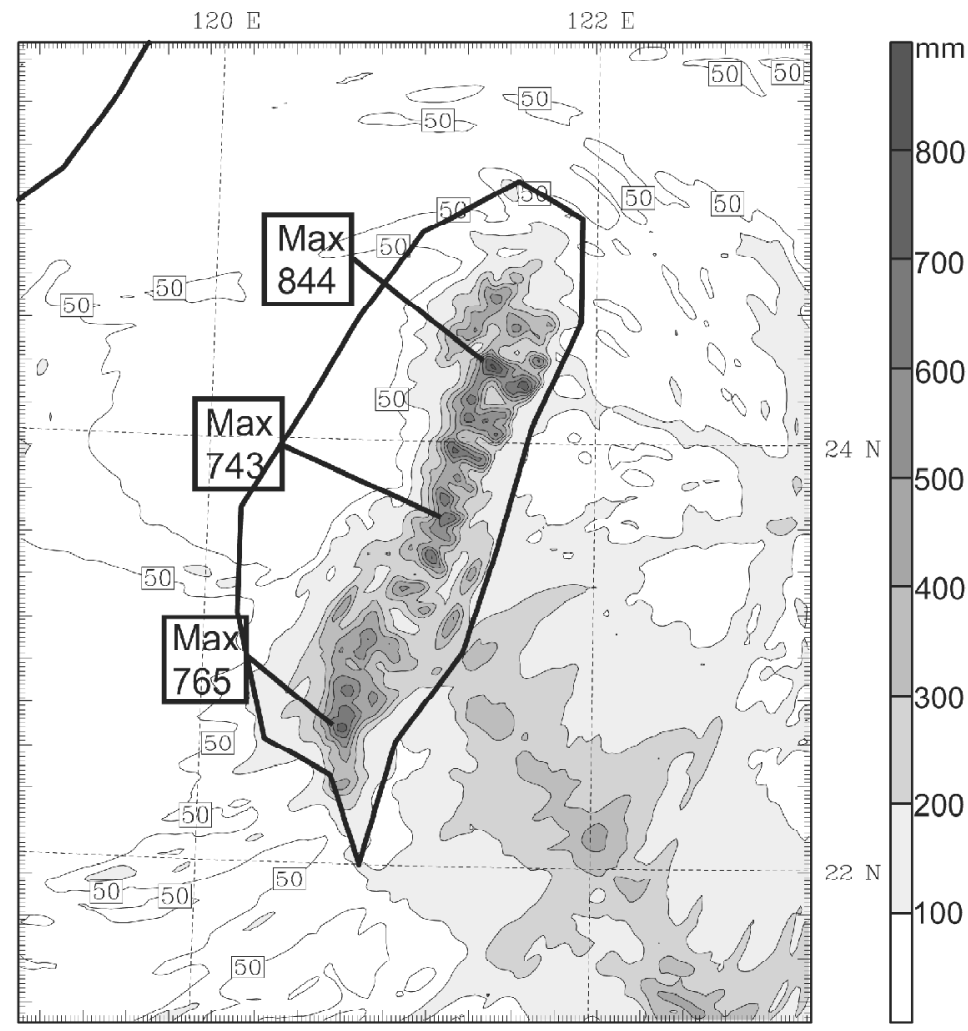

Fig. 10. 24-hour rainfall ending 0000 UTC 23 August 2000 for case CON2.

close to the observed maximum (Fig. 6). However, it was over $100 \mathrm{~mm}$ greater than observations. A similar magnitude of over-prediction was seen throughout the CMR north of the typhoon track. Predicted rainfall in the south was over-predicted even more. Model rainfall was 765 $\mathrm{mm}$, compared to observed rainfall of near $300 \mathrm{~mm}$. Despite the over-prediction, we will use CON2 to analyze the mechanisms which are responsible for producing extremely heavy rainfall during the passage of typhoon Bilis. Compared to the simulations of Lin et al. (2002) using COAMPS model without bogussing the vortex, the predicted rainfall amounts are much better.

The majority of the rainfall fell during the 6-hr period from 1200 to 1800 UTC 22 August, during which Bilis made landfall at approximately 1500 UTC. Depicted in Figs. 11a, b are the observed 3-hr precipitation amounts ending at 1500 UTC and 1800 UTC, respectively. Corresponding simulated amounts from CON2 are shown in Figs. 11c, d. The model track of Bilis and position at the end of the 3 hours is also shown in Fig. 11b. In both the observations and model predictions, a dipole pattern is readily apparent. Rainfall associated with the maximum north of the track is due to the easterly winds of the outer circulation to the north of the center impinging on the CMR. Near where the center of Bilis tracked, there is a relative minimum in rainfall, as the northerly winds associated with the forward part of the circulation would have 
been parallel to the CMR, and thus the rainfall would have been more difficult to be produced by orography. The southern maximum is associated with southwesterly winds on the south of the circulation, drawing air from the Taiwan Strait east into the south portion of the CMR. During 1200 - 1500 UTC, the CON2 predicted rainfall has the maxima in the north and the south of the CMR, and a minimum in between. Rainfall in the north is over predicted, generally by about $30-40 \mathrm{~mm}$ over the highest terrain. A partial explanation may be the lack of rain gauges over the highest terrain of the CMR. However, this fails to explain the over-prediction of rain in the south, by a factor of 2. During 1500 - 1800 UTC, the rainfall to the south of the track is over predicted by an even greater amount. Differences to the north of the track are approximately to the same degree as in the previous 3 hours.
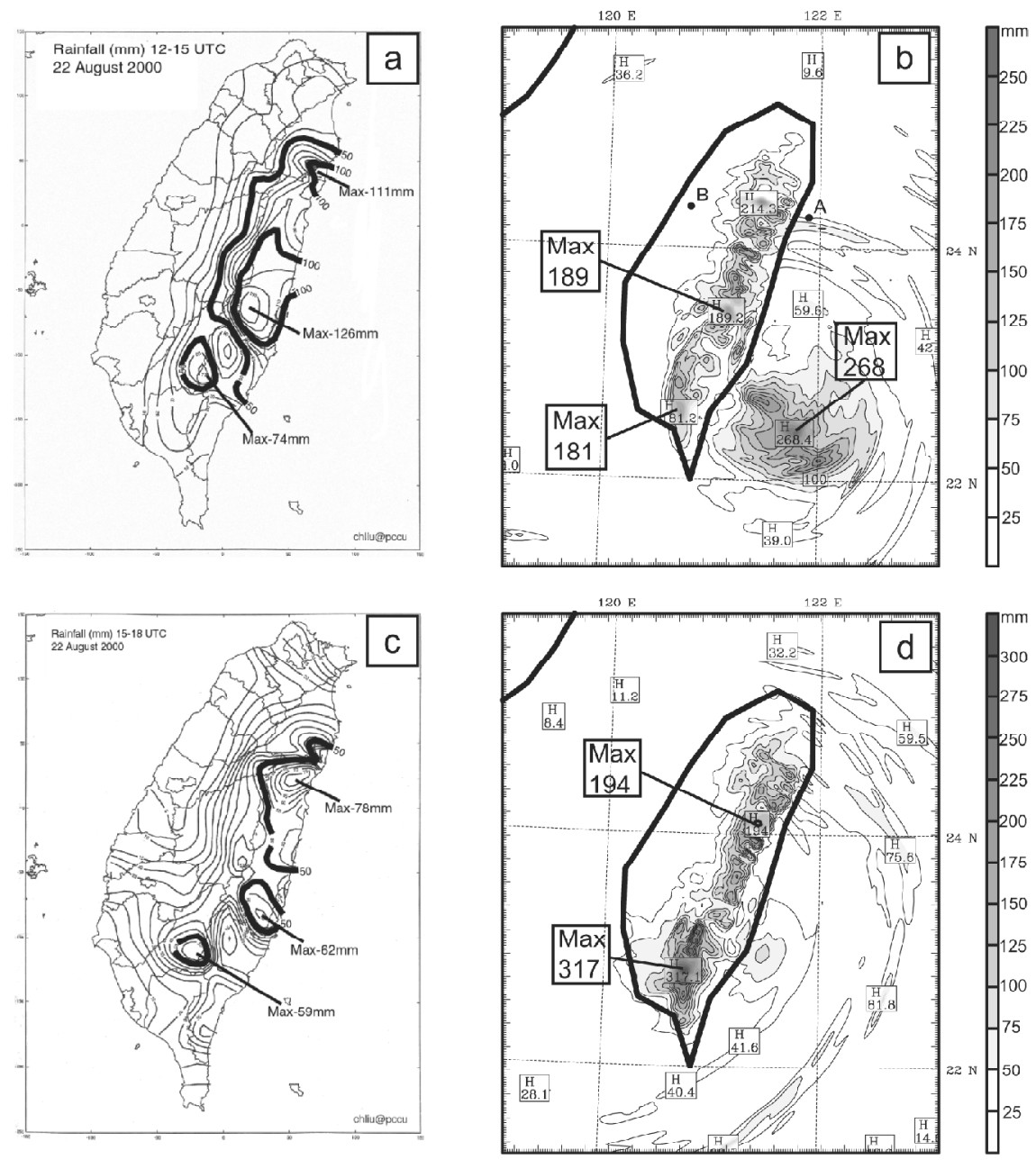

Fig. 11. 3-hour rainfall from observations (a) and (b) and case CON2 (c) and (d). (a) and (c) are from 12 - 15 UTC 22 August, (b) and (d) are from 15 - 18 UTC 22 August. 


\subsection{Common Ingredients for Heavy Orographic Rainfall Associated with Bilis (2000)}

The heavy rainfall can be attributed to a number of factors as proposed in Lin et al. (2001), which proposed that heavy orographic rainfall requires significant contributions from a set of common ingredients. One ingredient is steep topography, which is present all along the eastern side of Taiwan (Fig. 4b). The terrain rises from near sea level to elevations over $3 \mathrm{~km}$ in the CMR, with two peaks approaching $4 \mathrm{~km}$. The change in elevation is over a relatively short distance, yielding an average slope of $\sim 3 \mathrm{~km} / 80 \mathrm{~km}$. The CMR is an effective mechanism for lifting a low-level jet, another ingredient identified by Lin el al. (2001a). By their nature, typhoons contain low-level jets feeding air into the center of low-pressure, and the highest winds within tropical cyclones are found within the eyewall below $700 \mathrm{mb}$. Figure 12 shows $850 \mathrm{mb}$ geopotential height and windspeed at 1500 UTC 22 August. Within the eyewall winds exceed $70 \mathrm{~m} \mathrm{~s}^{-1}$, with winds above $30 \mathrm{~m} \mathrm{~s}^{-1}$ extending well away from the center. North of the landfall position, low-level winds within the circulation would slam into the high terrain of the CMR, inducing strong upward vertical motion, which is roughly proportional to $\mathbf{U d h} / \mathrm{dx}$, where $\mathbf{U}$ is the wind speed perpendicular to the mountain range, and $\mathrm{dh} / \mathrm{dx}$ is the slope of the mountain, in response to lower boundary forcing. The same process happened to the south of the track, where southwest winds impinged on the west side of the CMR.

Another ingredient is the high precipitation efficiency, which can be illustrated by the amount of rain that fell over the ocean, before the circulation interacted with Taiwan. Looking

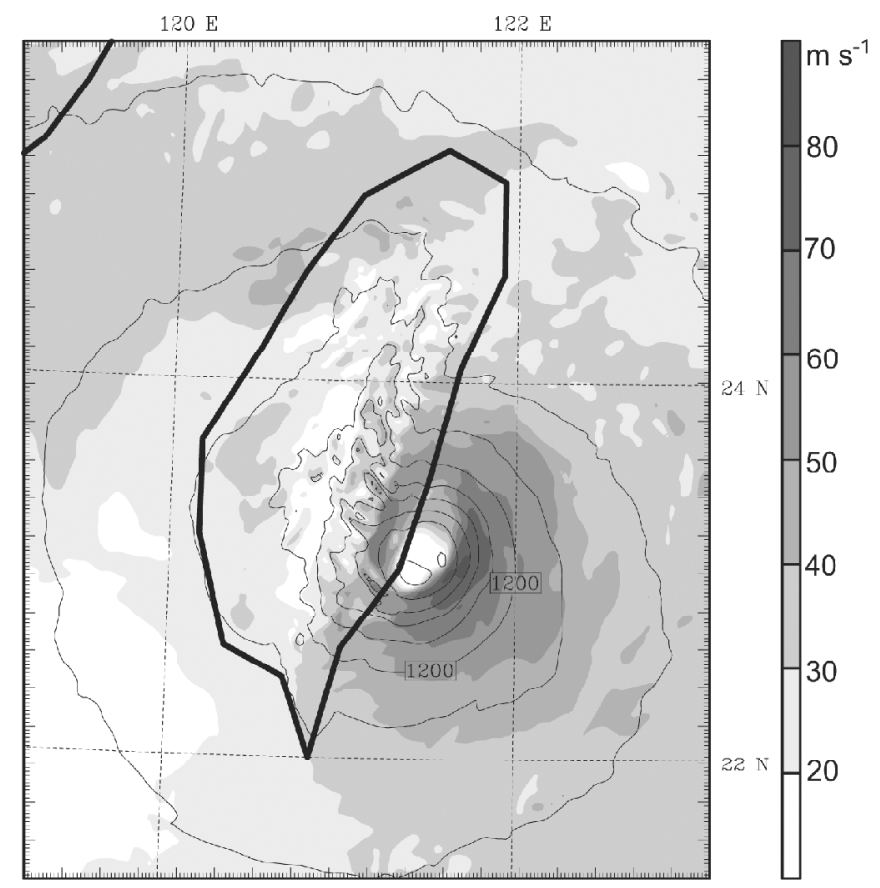

Fig. 12. $850 \mathrm{mb}$ geopotential height (contours) and windspeed (shaded, $\mathrm{m} \mathrm{s}^{-1}$ ) at 1500 UTC 22 August. 
at the 24-hour rainfall (Fig. 10), a widespread area of rainfall in excess of $100 \mathrm{~mm}$ over the open ocean is due to the overall circulation of the typhoon. An area in excess of $200 \mathrm{~mm}$, with peak amounts near $450 \mathrm{~mm}$, is present near the track of the typhoon, caused by the inner core of the circulation. Not surprisingly, high precipitable water values are present in the environment around the typhoon (Fig. 13). Areas to the east of Taiwan have precipitable water exceeding $70 \mathrm{~mm}$. Lin et al. (2001a) showed that high amounts of moisture upstream are a key ingredient for producing heavy rainfall, and this condition existed within the expansive circulation of Bilis. With lifting condensation levels (LCL) below $1000 \mathrm{~m}$ (Fig. 14), and a moist environment (Fig. 15), much of the precipitable water will be consumed as the airstream is advected over the CMR, which has peaks over $3000 \mathrm{~m}$.

A major factor in the production of heavy rainfall was the existence of a conditionally and/or potentially unstable environment within the typhoon circulation. When the air is forced up the CMR as the typhoon comes ashore, heavy amounts of rainfall are inevitable. Essentially, there are two different types of soundings within the circulation of Bilis: soundings that have been modified by convection, especially within the inner core of the typhoon, and soundings further away from the center modified less or not at all by convection. This distribution can be seen in Fig. 16, which show CAPE at 1500 UTC. Near the center and around the prominent feeder bands, the CAPE is very low. Away from convective modification, the CAPE approaches $1000 \mathrm{~J} \mathrm{~kg}^{-1}$. Noteworthy features are the stream of higher values of CAPE impinging on the north central portion of the CMR during this entire feature. Also note the higher CAPE impinging on the south CMR from the southwest. This seems to have been a factor in the model producing such high amounts of rainfall in the southern CMR.

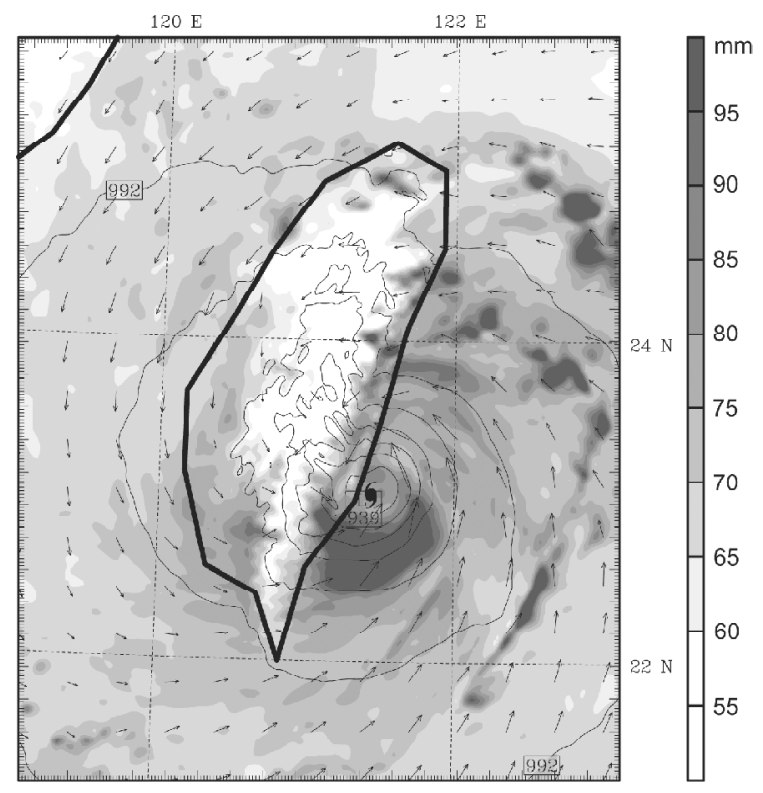

Fig. 13. Sea level pressure (contours) and precipitable water (shaded, mm) at 1500 UTC 22 August. 


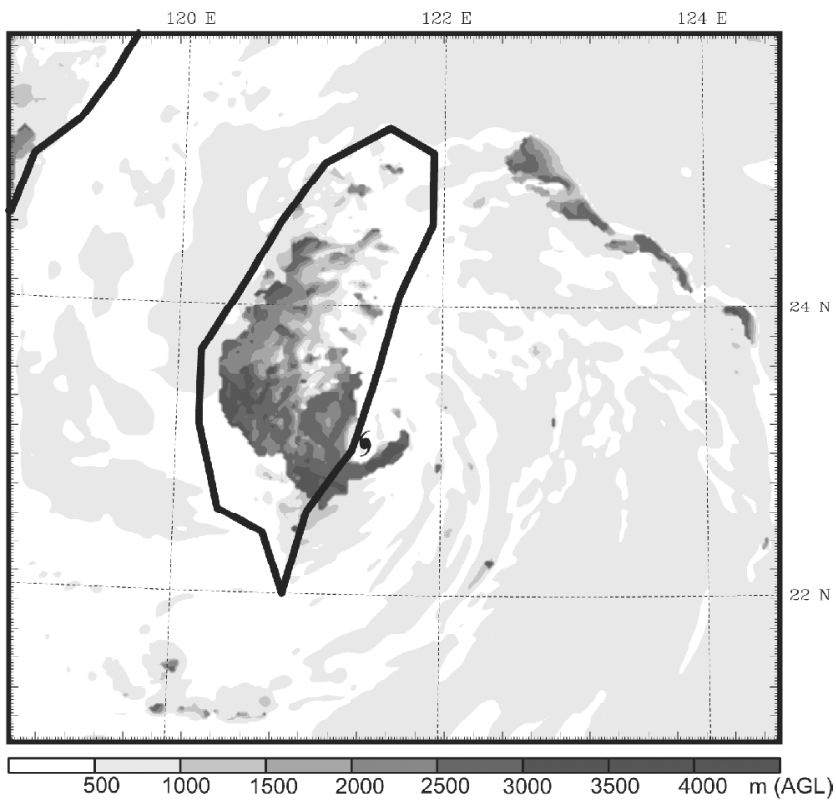

Fig. 14. Lifting condensation level (LCL, m) at 1500 UTC 22 August.

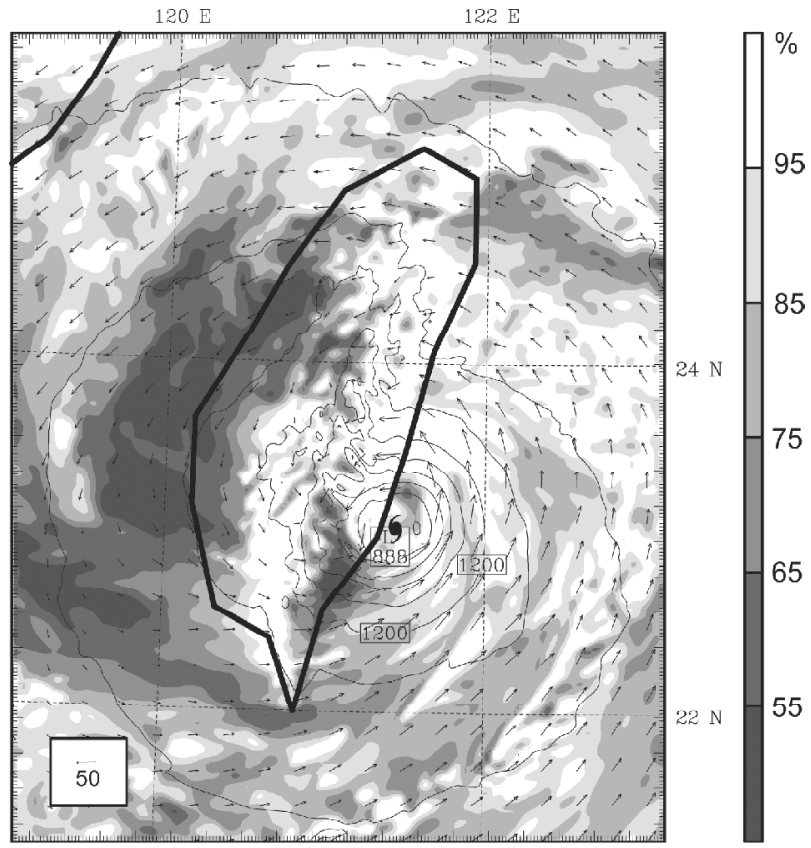

Fig. 15. $850 \mathrm{mb}$ geopotential height (contours) and relative humidity (shaded) at 1500 UTC 22 August. 
The parameter $\mathrm{d} \theta_{\mathrm{e}} / \mathrm{dz}$ can signal convective instability when values of $\mathrm{d} \theta_{\mathrm{e}} / \mathrm{dz}<0$. Figure 17 shows $\mathrm{d} \theta_{\mathrm{e}} / \mathrm{dz}$ at $\sigma=.95$, for 1500 UTC 22 August. Calculations are made at levels immediately adjacent to $\sigma=.95$. Very low values are present upstream and east of the central CMR, and these values would have been advected straight into the Mt. Jade area. In the area upstream and west of southern Taiwan, there is an area of very large negative $\mathrm{d} \theta_{\mathrm{e}} / \mathrm{dz}$, collocated with the area of high CAPE mentioned previously. The source of this conditionally (high CAPE) and convectively (negative $\mathrm{d} \theta_{\mathrm{e}} / \mathrm{dz}$ ) unstable air appears to be in the lee of Taiwan, and is a product of the airstream impinging on and flowing over the northern CMR. This air to the west of the CMR is initially too dry $(\mathrm{RH}<60 \%)$ to produce convection and rainfall, but is able to pick up enough moisture (presumably from the ocean) to produce the rainfall in the southern CMR. The Blackadar PBL parameterization may have moistened the boundary layer too quickly, resulting in the drastic overprediction to the south of the track.

Figure 18a is a sounding away from the inner core, just off the northeastern coast of Taiwan (point A, Fig. 11c), for 22 August 15 UTC. The model sounding is quite moist and conditionally unstable. In fact, there exists a deep layer which behaves like a moist absolutely unstable layer (MAUL) (Bryan and Fritsch 2000). However, this MAUL may be a result of the lack of a cumulus scheme in the 7-km domain, which would result in artificially unstable air being fed into the inner domain. The lifting condensation level (LCL) was $927 \mathrm{mb}$ and the level of free convection was at $928 \mathrm{mb}$. The CAPE was $980 \mathrm{~J} \mathrm{~kg}^{-1}$, not much higher than CAPE from an observed sounding from ROIG (Fig. 19), approximately $300 \mathrm{~km}$ east northeast

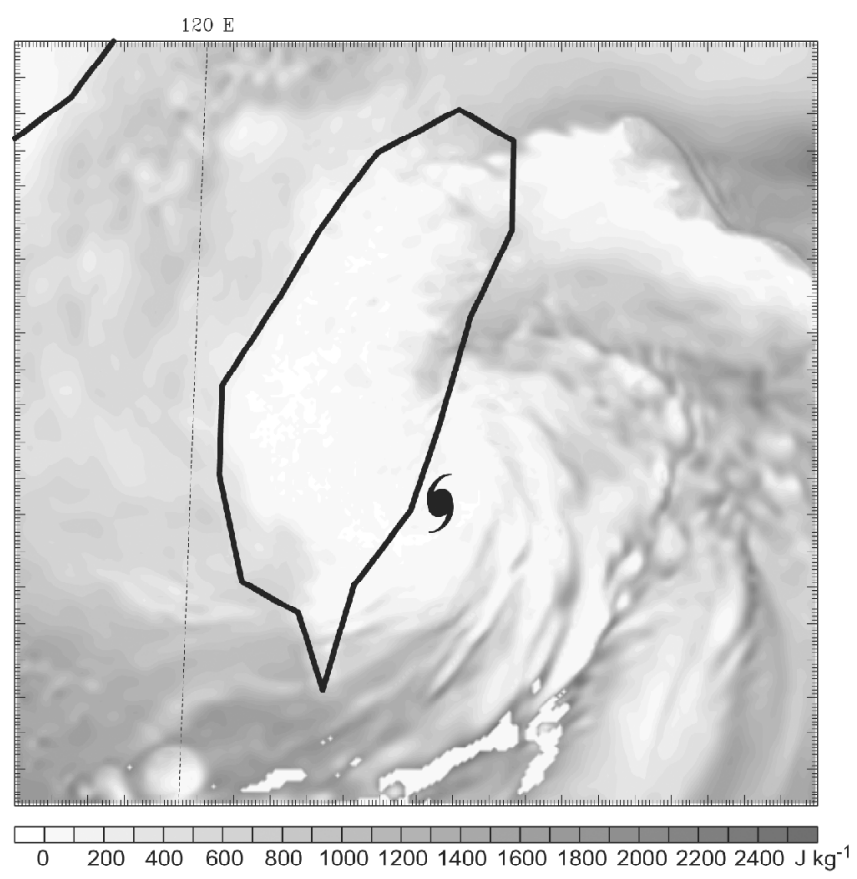

Fig. 16. Convectively Available Potential Energy (CAPE, $\mathrm{J} \mathrm{kg}^{-1}$ ) at $1500 \mathrm{UTC}$ 22 August 2000. 


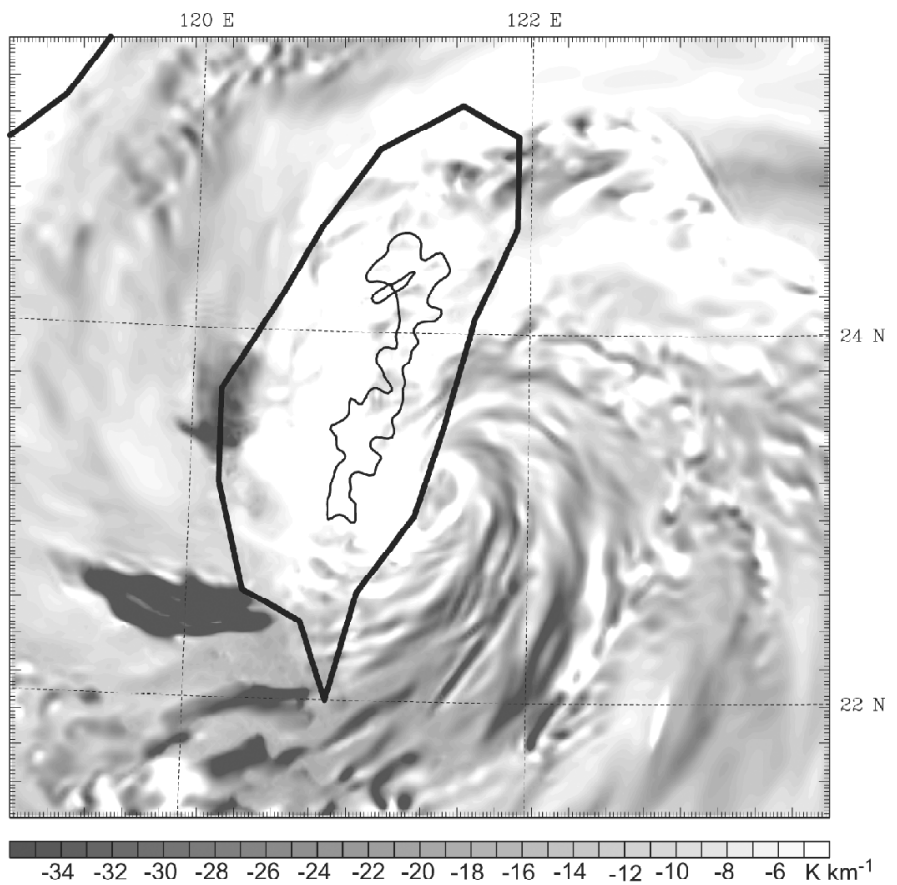

Fig. 17. Distribution of $\mathrm{d} \theta_{\mathrm{e}} / \mathrm{dz}\left(\mathrm{K} \mathrm{km}^{-1}\right)$ at $\sigma=.95$, computed from adjacent sigma levels, at 1500 UTC 22 August 2000.

of the northern tip of Taiwan. However, amounts of CAPE were not significantly different in separate the cumulus parameterization sensitivity experiments at 7-km grid spacing (Witcraft 2004), suggesting much of the instability seen in the model results was real. The winds in the outer circulation average around in excess of $30 \mathrm{~m} \mathrm{~s}^{-1}$ in the lower levels, and should allow most of this conditional instability to be realized as the northern side of the circulation impinges on the CMR. By comparison, Figure $18 \mathrm{~b}$ is a model sounding from the western side of the CMR (point B, Fig. 11c), where the airflow is modified by the CMR. A prominent pool of warm air near the surface has been created by the adiabatic warming as the air crossed over the CMR and descends. The LCL is $763 \mathrm{mb}$, due to both the adiabatic warming and the consumption of water vapor as it was condensed into clouds and precipitation over the CMR. This also leads to very low CAPE, almost near $0 \mathrm{~J} \mathrm{~kg}^{-1}$. The adiabatic warming and downward motion in the lee of the CMR cause a sharp gradient in heavy rainfall in the lee of the mountains, especially in northwest Taiwan (Fig. 11). Rainfall in this area was generally $50 \mathrm{~mm}$ or less. After impinging on the CMR, the air rises, and cools until saturation. Moisture in the air condenses into cloud and liquid water below the freezing level, while it is converted or deposited as cloud ice, snow, and graupel above the freezing level. This releases large amounts of latent heat into the air. The air warms further from adiabatic downsloping as it descends the $\mathrm{CMR}$, and dries out dramatically. Relative humidity $(\mathrm{RH})$ drops below $65 \%$ in a large area to the west of the CMR (Fig. 15). An area of lower RH is also present to the south of the landfall 

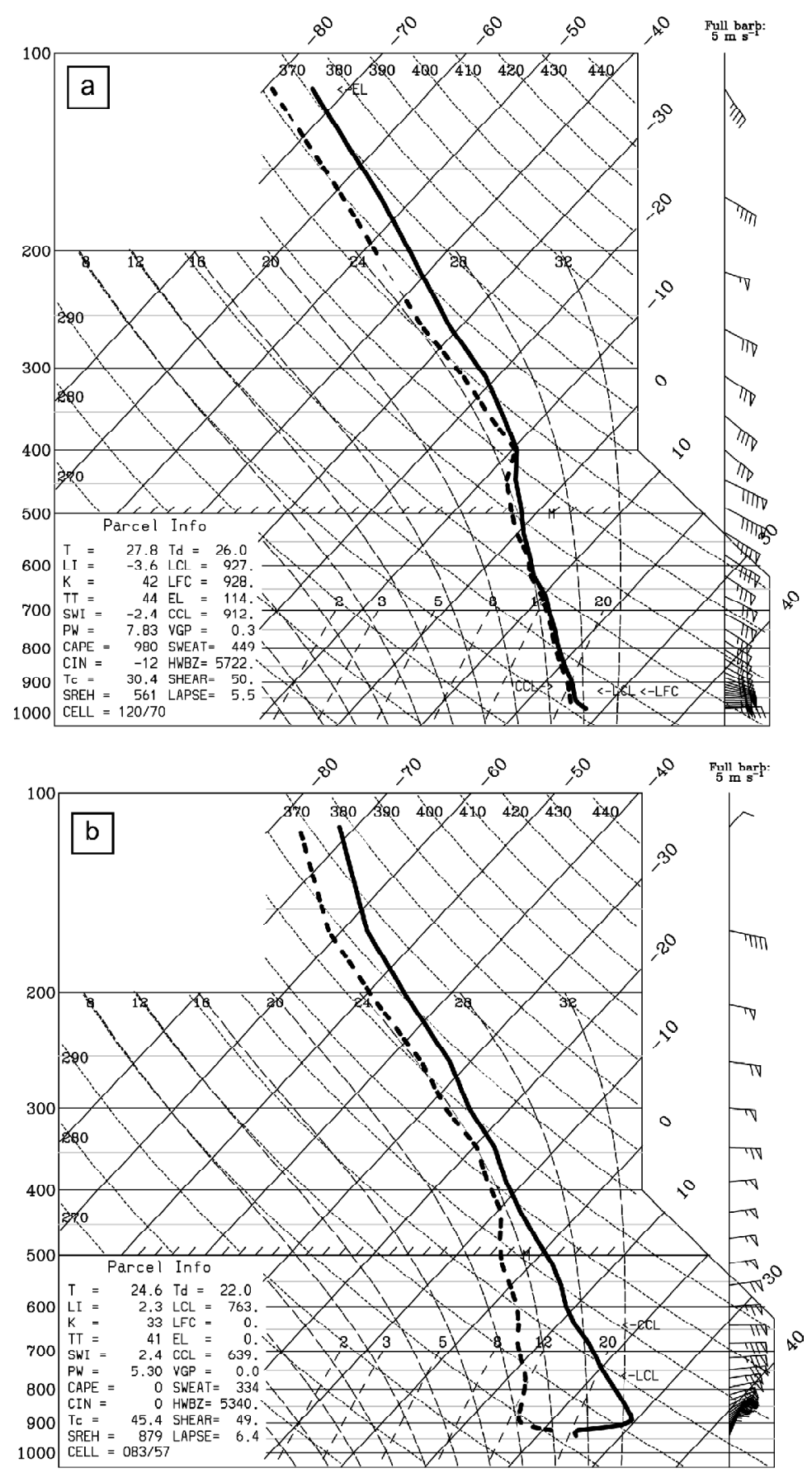

Fig. 18. (a) Model sounding to the east of the CMR, and (b) Model sounding to the west of CMR, both at 1500 UTC 22 August 2000. 
position, i.e., to the southeast of the CMR. These areas are not conducive to the formation of any significant rainfall, due to descending motion. In fact, dry air entrainment, from the downsloping and adiabatic warming off the southeast CMR, appears to have helped weaken Bilis as it approached Taiwan.

Another process acting to mitigate rainfall is the formation of mountain waves. Figure 20 shows a series of cross sections through the northern CMR, where the most intense mountain wave activity exists. At 1500 UTC 22 August (Fig. 20a), Bilis is making landfall, with the most intense winds impinging on the CMR at that time. Deep clouds are present on the eastern slopes of the CMR, with shallower clouds to the east of the CMR over the Pacific Ocean. The latent heat release within the clouds, combined with the strong winds, leads to the formation of an intense mountain wave, creating strong downward motion on the west side of the CMR (Raymond 1972; Fraser 1973, Barcilon et al. 1979, Lin et al. 1998). This leads to an abrupt warming and drying of the airstream, hence the sharp gradient in precipitation to the west of the CMR. The environment in the area remains relatively unchanged $3 \mathrm{hrs}$ later (Fig. 20b), with deep clouds now isolated to the upslope areas. New mountain waves also have been generated downstream of the CMR due to the westward propagation of Bilis.

\subsection{The Use of Moisture Flux for Estimating Rainfall and the Relevant Dynamics}

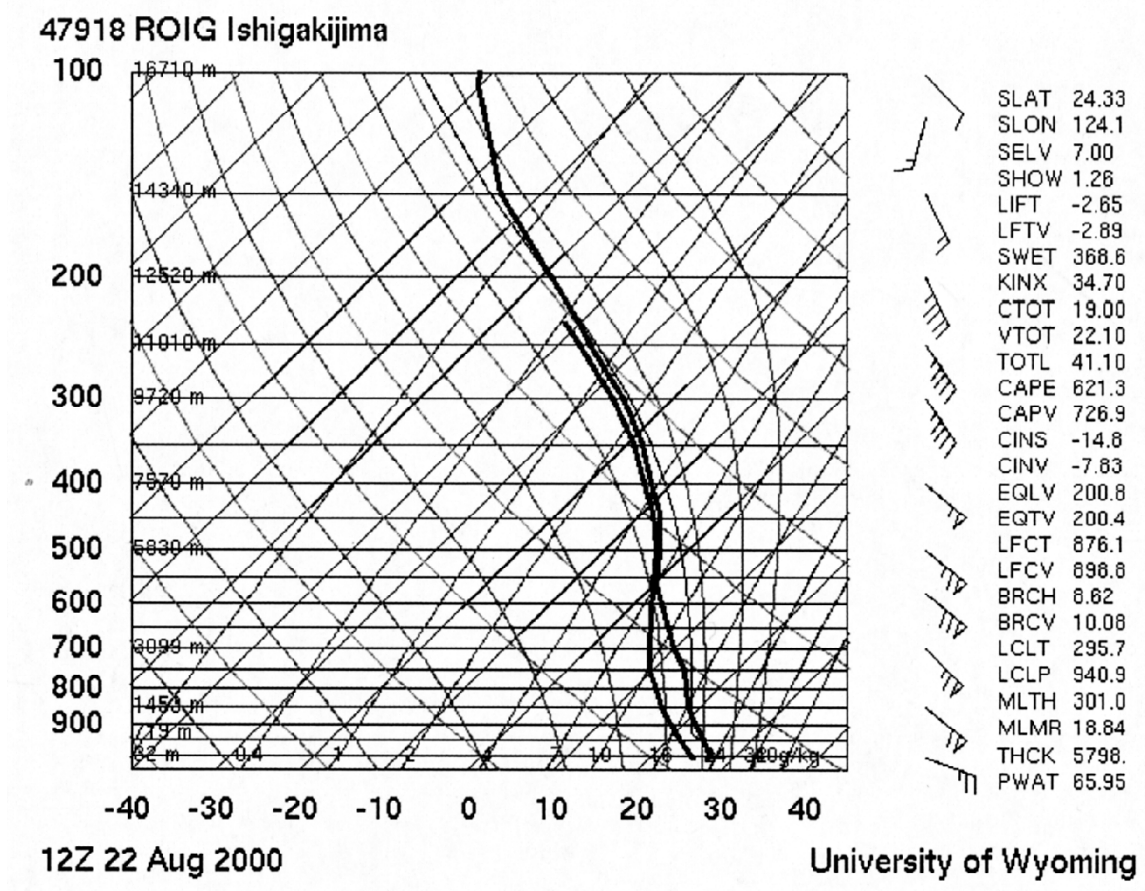

Fig. 19. Actual RAOB sounding from Ishigakijima, Japan, at 1200 UTC $22 \mathrm{Au}-$ gust 2000. 

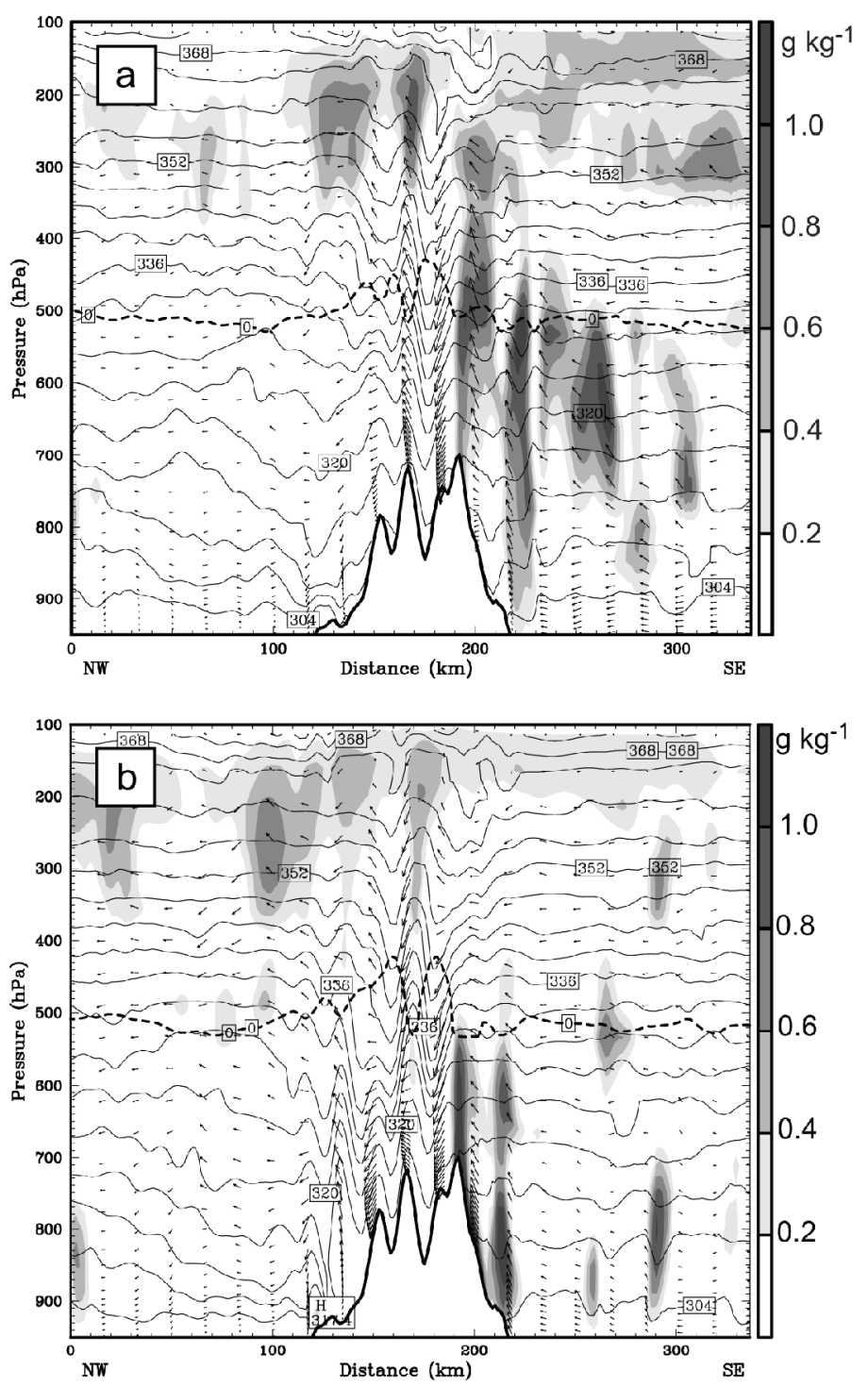

Fig. 20. Vertical cross section over the central CMR. Shown are cloud mixing ratio (shaded, $\mathrm{g} \mathrm{kg}^{-1}$ ), potential temperature (contours, $\mathrm{K}$ ), freezing level (dashed line), and circulation vectors at (a) 1500 UTC, and (b) 1800 UTC 22 August 2000. Cross section location is shown in Fig. 11c.

To help understand the processes involved in orographic rain formation, we use the finest resolution model output to examine the orographic precipitation efficiency formula proposed by Lin et al. (2001). From their Eq. (8), the total precipitation P may be estimated by: 


$$
P=\left(\rho / \rho_{w}\right) E\left[V_{H} \cdot \nabla h+w_{e n v}\right] q L_{s} / c_{s}
$$

where $V_{H} \cdot \nabla h$ is the vertical motion induced by the horizontal winds impinging on orography (Doswell et al. 1998; Lin et al. 2001), $w_{e n v}$ is the environmentally forced vertical motion, $q$ is the water vapor mixing ratio, $\rho$ and $\rho_{w}$ are the air density $\left(\sim 1 \mathrm{~kg} \mathrm{~m}^{-3}\right)$ and water density $\left(\sim 1000 \mathrm{~kg} \mathrm{~m}^{-3}\right)$ respectively, $c_{s}$ is the propagation speed of the convective system, and $L_{s}$ is the horizontal scale of the mountain. The precipitation efficiency $(E)$ is defined as the ratio of precipitation water mass over the water mass inflow (Frankhauser 1988; Doswell et al. 1996). The value of $E$ is difficult to obtain, as it depends on microphysical processes (such as evaporation of hydrometeors during descent) and advective processes (such as ice particles escaping through outflow). In addition, rapid vertical motions at the convective scale can make this number ambiguous at best. Both $L_{s}$ and $c_{s}$ can be estimated, but are too sensitive to be taken into account in Eq. (1). Thus, the precipitation $\mathrm{P}$ may be roughly regarded as a function of both the orographically induced vertical motion and/or the environmentally-induced vertical motion, each multiplied by the mixing ratio. This will allow us to determine how much of the heavy rainfall over the CMR can be attributed to the typhoon, and how much is from orographic processes.

Figure 21 shows the orographically induced vertical moisture flux, $(\boldsymbol{V} \cdot \nabla h) q$, and the general moisture flux, $w q$, estimated from CON-2 from 1500-1800 UTC 22 August. The simulated wind and water vapor fields are used for these calculations. For both fluxes, an elongated positive area exists along the CMR, with several maxima associated the highest terrain. Over the ocean, lower values of general moisture flux $(w q)$ correlate well with the same areas where the model produced rainfall. These areas are mainly caused by lower level convergence, and are associated with the eye wall and the spiral rain bands. The highest areas general moisture flux and orographic moisture flux match very well, meaning that orographically induced vertical motions were largely responsible for the heavy rainfall over Taiwan.

\subsection{A simple Moisture Budget Analysis}

In order to understand the dynamics of the formation of orographic precipitation, we perform a moisture budget analysis. By ignoring the detailed microphysical processes, the moisture budget may be derived from the conservation equations of water vapor and liquid water,

$$
M_{v}+M_{l}+E=R+S_{q v}+S_{q l},
$$

where $M_{v}$ and $M_{l}$ are the convergence of water vapor and liquid water, respectively, $E$ the surface evaporation rate, $\mathrm{R}$ is the precipitation rate, and $S_{q v}$ and $S_{q l}$ are the storages of water vapor and liquid water, respectively. If we assume $M_{l}$ is much less than $M_{v}$, and $E, S_{q v}$, and $S_{q l}$ are negligible, then the total precipitation can be estimated by:

$$
P=D R \approx D M_{v}=-\frac{D}{g} \int_{0}^{p_{s}} \nabla \cdot\left(q_{v} \boldsymbol{V}\right) d p
$$



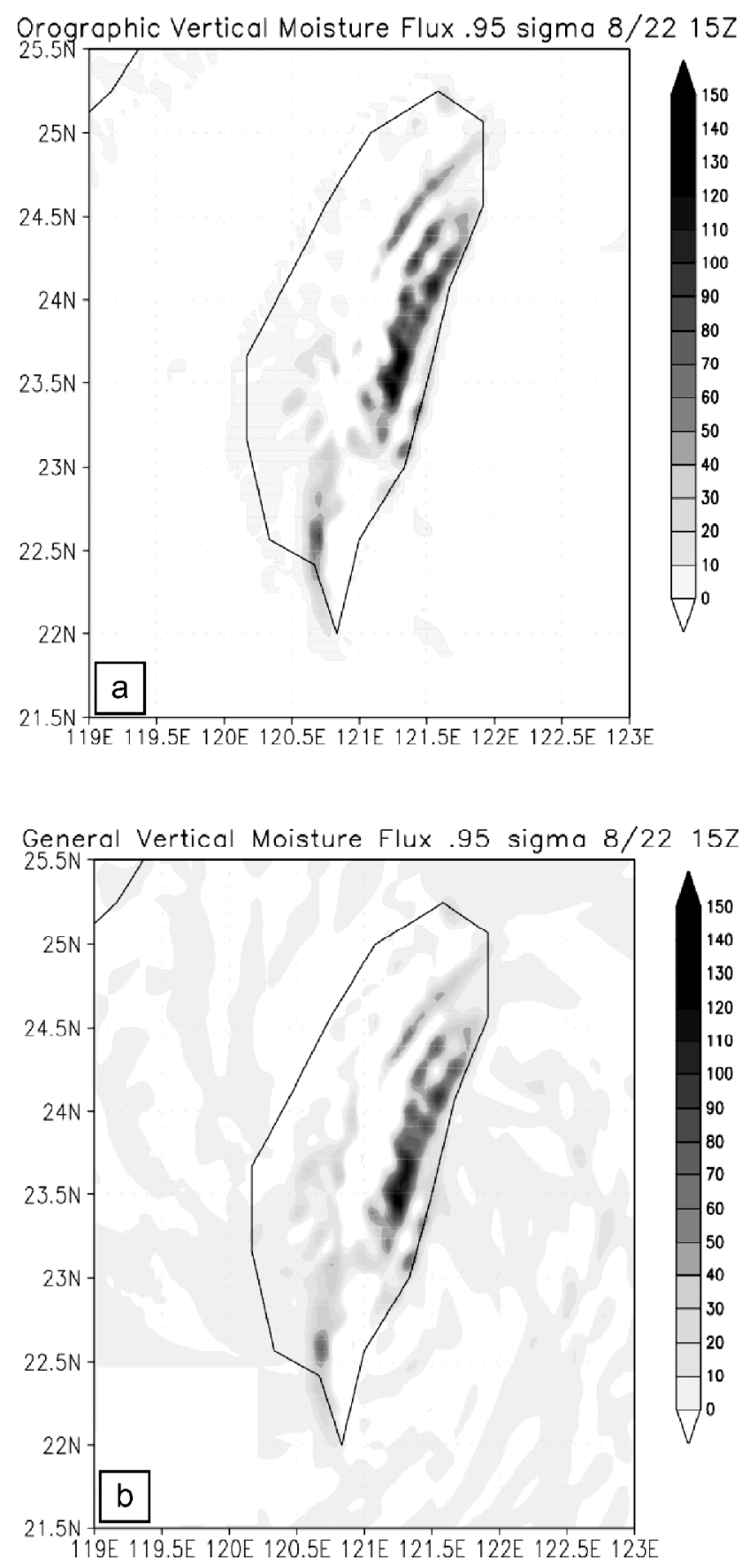

Fig. 21. Orographically induced vertical moisture flux, $(\boldsymbol{V} \cdot \nabla h) q($ a), and the general vertical moisture flux $(w q)(b)$, valid at 1500 UTC 22 August 2000, based on CON2 with 2.33-km grid simulation. 
where $D$ is the duration of precipitation and $p_{s}$ is the surface pressure. Thus, the precipitation can be estimated from model results,

$$
P=-\frac{D}{g} \int_{p_{r}}^{p_{s}}(\nabla \cdot \boldsymbol{V}) q_{v} d p-\frac{D}{g} \int_{p_{r}}^{p_{s}}\left(\boldsymbol{V} \cdot \nabla q_{v}\right) d p
$$

A

$\mathrm{B}$

where $p_{t}$ is the pressure at the top of the model domain. Physically, the first term (Term A) on the right side represents the moisture convergence induced by flow divergence, while the second term (Term B) represents the moisture convergence induced by moisture advection.

Figure 22 shows positive areas of Term $A$, Term $B$, and $P$ of Eq. (4) from Bilis simulation results at 1500 UTC 22 August. The results indicate that the positive areas of $P$ (Fig. 22a) estimated for $3 \mathrm{hrs}$ by the above moisture budget is similar to the observed rainfall (Fig. 6) and model simulated patterns and amounts (Fig. 11). Term A (Fig. 22b) indicates that moisture convergence produced by flow convergence is dominant over the upslope of the CMR, while that produced by moisture advection is dominant over the lee side of the CMR. The pattern of the precipitation $(P)$ is similar to Term $A$, which implies that the generation of upslope orographic rainfall during the passage of a TC over a mesoscale mountain is controlled by the orographically forced convergence. In addition, comparing $P$ with the orographic and general moisture fluxes calculated at lower level (Fig. 21) also implies that the upslope orographic precipitation is dominated by lower-level convergence.

\section{CONCLUDING REMARKS}

In this study, we used a nonhydrostatic mesoscale model (MM5) to simulate Supertyphoon Bilis (2000) to study orographic rainfall over the Central Mountain Range (CMR) of Taiwan. In the first portion we establish a control simulation with $2.33-\mathrm{km}$ grid spacing to investigate the physical mechanisms responsible for heavy orographic rainfall. For the second portion, we investigate sensitivity of track, strength, and rainfall for various precipitation physics and 2 different model grid spacing. It is critical to attain a good forecast track to properly simulate the rainfall.

The orographic rainfall prediction improved as grid spacing decreased. At $21 \mathrm{~km}$, rainfall was approximately half of observations. The rainfall maximum at $7 \mathrm{~km}$ was near the observed values, and there was a $10-15 \%$ over-prediction of rainfall at $2.33-\mathrm{km}$ grid spacing. An analysis of the $2.33-\mathrm{km}$ control simulation reveals that nearly all the common ingredients for heavy orographic rainfall identified by Lin et al. (2001) were met. A low-level jet associated with the northern circulation of Bilis impinged on the steep topography of the CMR, and induce strong upward vertical motion. The environment in and around Bilis contained high moisture content, another key ingredient. The environment was also conditionally unstable, with areas of CAPE approaching $1000 \mathrm{~J} \mathrm{~kg}^{-1}$, and potentially (convectively) unstable, with $\mathrm{d} \theta_{\mathrm{e}} / \mathrm{dz}<0$. The circulation of Bilis was ripe for the production of heavy rainfall. 

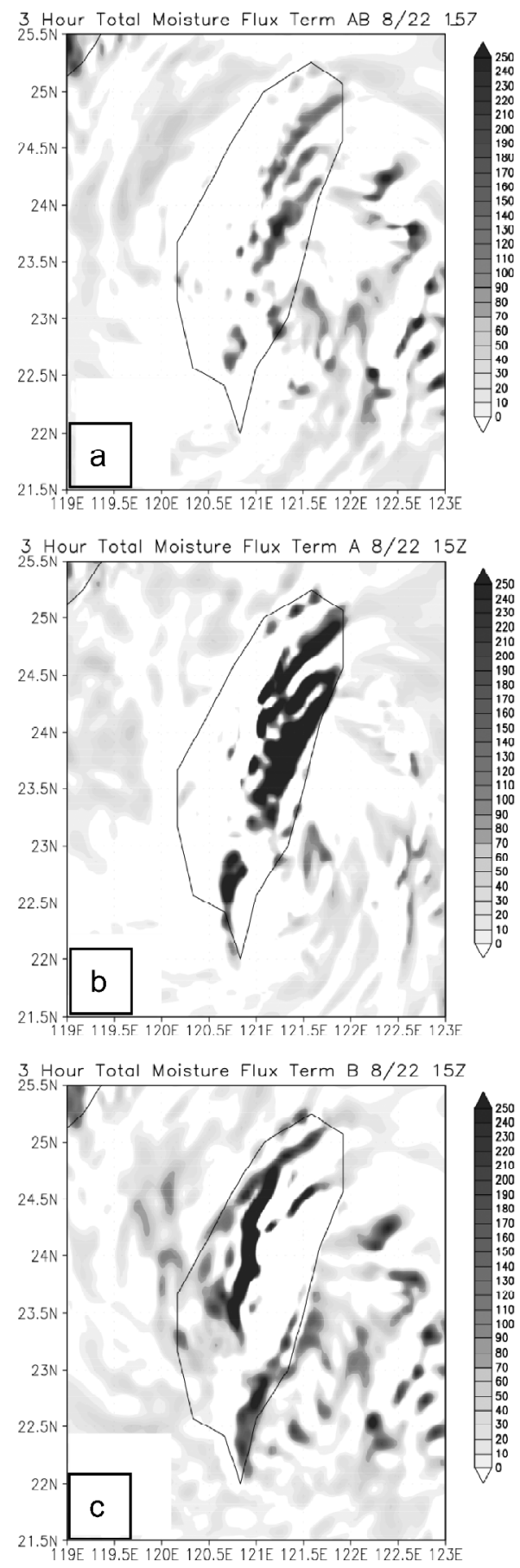

Fig. 22. Term $P$ (a), term $A$ (b), and term $B$ (c) of Eq. (4) for 3 hrs ending 1500 UTC 22 August 2000 from case CON2. 
A moisture flux model was used to estimate rainfall and the relevant dynamics. The orographically induced vertical moisture flux, $(\boldsymbol{V} \cdot \nabla h) q$, was able to provide rainfall estimates that compared well with observations. By comparing the results with the general moisture flux, $w q$, it was shown that a large portion of the rainfall over the mountains was from orographically induced vertical motions. A simple moisture budget, Eq. (4), was used to better understand the dynamics of orographic precipitation. It was shown that the vertical moisture flux was mainly induced by flow convergence in the upslope region, while it was mainly induced by moisture advection on the lee side of the CMR and over the ocean. These terms largely cancel, but the remainder [term P, Eq. (4)] is similar to term A, indicating that the orographic rainfall is largely due to orographically induced convergence.

We have also performed a sensitivity study of Bilis using various cumulus and microphysical parameterizations (Witcraft 2004). Rainfall, track, and wind speed were highly sensitive to the choice of cumulus parameterization. Tests of four different microphysical parameterization (grid-explicit) schemes at 7-km grid spacing with no cumulus parameterization reveal that rainfall, as well as the strength and track, were not very sensitive to the microphysical scheme. These results are consistent with other studies by Davis and Bosart (2001) and Kuo et al. (1996). Additional factors can influence the simulation of TCs. The intensity a structure has been shown to be highly sensitive to the choice of PBL scheme (Braun and Tau 2000; Davis and Bosart 2001). Nearly all of the simulations over predicted rainfall in the south, possibly due to the Blackadar PBL scheme being too quick to moisten the boundary layer after traversing the CMR. We would like to try other PBL schemes to see if rainfall in the south can be reduced. Another major factor is the initial data. Unlike in the Atlantic, reconnaissance aircraft for the most part have not flown into TCs. However, the government of Taiwan has initiated the DOTSTAR (Dropsonde Observations for Typhoon Surveillance near the Taiwan Region) program, which will fly into the outer circulations of typhoons threatening Taiwan. Future studies can determine how much this reconnaissance data improves the model predictions. Another area of improvement is in the vortex bogussing. In most of the simulations, the TC weakened significantly from its specified strength. A new scheme needs to be developed that is more physically consistent with the model.

Acknowledgments The authors would like to thank Drs. M. Kaplan, G. Lackmann, and F. Semazzi of NC State University, C. Y. Huang of National Central University and C. C. Wu of National Taiwan University for their helpful discussions and comments. This research is supported by the NSF UCAR Cooperative Agreement ATM-9732665 and NSF Grant ATM0096876."

\section{REFERENCES}

Alpert, P., 1986: Mesoscale indexing of the distribution of orographic precipitation over high mountains. J. Clim. Appl. Meteor., 25, 532-545.

Anthes, R. A., E. Y. Hsie, and Y. H. Kuo, 1987: Description of the Penn State/NCAR Mesoscale Model Version 4 (MM4). NCAR/TN-282+STR, National Center for Atmospheric Research, Boulder, CO, 66 pp. 
Barcilon, A., J. C. Jusem, and P. G. Drazin, 1979: On the two-dimensional, hydrostatic flow of a stream of moist air over a mountain ridge. Geophys., Astrophys., Fluid Dynamics, 12, 1-16.

Betts, A. K., and M. J. Miller, 1986: A new convective adjustment scheme. Part I: Observational and Theoretical Basis. Quart. J. Roy. Soc., 112, 677-691.

Betts, A. K., and M. J. Miller, 1993: The Betts-Miller scheme. The Representation of Cumulus Convection in Numerical Models, Meteorological Monographs. Am. Meteor. Soc., 46, 107-121.

Braun, S. A., and W. K. Tao, 2000: Sensitivity of High-Resolution Simulations of Hurricane Bob (1991) to Planetary Boundary Layer Parameterizations. Mon. Wea. Rev., 128, 39413961.

Bryan, G. H., and M. J. Fritsch, 2000: Moist Absolute Instability: The Sixth Static Stability State. Bull. Am. Meteo. Soc., 81, 1207-1230.

Chiao, S., and Y. L. Lin, 2003: Numerical Modeling of an Orographically Enhanced Precipitation Event Associated with Tropical Storm Rachel over Taiwan. Wea. For., 18, 325344.

Davis, C., and L. F. Bosart, 2002: Numerical Simulations of the Genesis of Hurricane Diana (1984). Part II: Sensitivity of Track and Intensity Prediction. Mon. Wea. Rev., 130, 1100-1124.

Davis, C., and S. Low-Nam, 2001: The NCAR-AFWA tropical cyclone bogussing scheme. A report prepared for the Air Force Weather Agency (AFWA), 12 pp.

Fraser, A. B., R. C. Easter, and P. V. Hobbs, 1973: A Theoretical Study of the Flow of Air and Fallout of Solid Precipitation Over Mountainous Terrain: Part I. Airflow Model.J. Atmos. Sci., 30, 801-812.

Grell, G. A., J. Duhdia, and D. R. Stauffer, 1994: A Description of the Fifth-Generation Penn State/NCAR Mesoscale Model (MM5). NCAR Tech. Note 398, 121 pp.

Kuo, Y. H., R. J. Reed, and Y. Liu, 1996: The ERICA IOP 5 Storm. Part III: Mesoscale Cyclogenesis and Precipitation Parameterization. Mon. Wea. Rev., 124, 1409-1434.

Lin, Y. L., 1993: Orographic effects on airflow and mesoscale weather systems over Taiwan. Terre. Atmos. Ocean. Sci., 4, 381-420.

Lin, Y. L., R. D. Farley, and H. D. Orville, 1983: Bulk Parameterization of the Snow Field in a Cloud Model. J. Appl. Meteo., 22, 1065-1092.

Lin, Y. L., J. Han, Hamilton, D. W. Huang, and Ching-Yuang. 1999: Orographic Influence on a Drifting Cyclone. J. Atmos. Sci., 56, 534-562.

Lin, Y. L., S. Chiao, T. A. Wang, M. L. Kaplan, and R. P. Weglarz, 2001: Some Common Ingredients for Heavy Orographic Rainfall. Wea. For., 16, 633-660.

Lin, Y. L., D. B. Ensley, S. Chiao, and C. Y. Huang, 2002: Orographic Influences on Rainfall and Track Deflection Associated with the Passage of a Tropical Cyclone.Mon. Wea. Rev., 130, 2929-2950.

Liu, Y., D. L. Zhang, and M. K. Yau, 1997: A Multiscale Numerical Study of Hurricane Andrew (1992). Part I: Explicit Simulation and Verification. Mon. Wea. Rev., 125, 3073-3093. 
Low-Nam, S., and C. Davis, 2001: Development of a tropical cyclone bogussing scheme for the MM5 system. Preprint, The Eleventh PSU/NCAR Mesoscale Model Users' Workshop, June 25-27, 2001, Boulder, Colorado, 130-134.

Raymond, D. J. 1972: Calculation of Airflow Over an Arbitrary Ridge Including Diabatic Heating and Cooling. J. Atmos. Sci., 29, 837-843.

Smith, R. B., and D. F. Smith, 1995: Pseudoinviscid Wake Formation by Mountains in Shallow-Water Flow with a Drifting Vortex. J. Atmos. Sci., 52, 436-454.

Tao, W. K., and J. Simpson, 1993: The Goddard cumulus ensemble mode. Part I: Model Description. Terr., Atmos. Ocean.. Sci., 4, 35-72.

Tripoli, G. J., 1992: An explicit three-dimensional nonhydrostatic numerical simulation of a tropical cyclone. Meteor. Atmos. Phys., 49, 229-254.

Wang, W., and N. L. Seaman, 1997: A Comparison Study of Convective Parameterization Schemes in a Mesoscale Model. Mon. Wea. Rev., 125, 252-278.

Weisman, M. L., W. C. Skamarock, and J. B. Klemp, 1997: The Resolution Dependence of Explicitly Modeled Convective Systems. Mon. Wea. Rev., 125, 527-548.

Witcraft, N. C., 2004: The dynamics of orographic rainfall and track deflection associated with the passage of a tropical cyclone over a mesoscale mountain. M. S. Thesis, North Carolina State Univ., N. C., 188 pp.

Wu, C. C., 2001: Numerical Simulation of Typhoon Gladys (1994) and Its Interaction with Taiwan Terrain Using the GFDL Hurricane Model. Mon. Wea. Rev., 129, 1533-1549.

Wu, C. C., T. H. Yen, Y. H. Kuo, and W. Wang, 2002: Rainfall Simulation Associated with Typhoon Herb (1996) near Taiwan. Part I: The Topographic Effect. Wea. For., 17, 1001-1015.

Zhang, D. L., and R. A. Anthes, 1982: A High-Resolution Model of the Planetary Boundary Layer-Sensitivity Tests and Comparisons with SESAME-79 Data.J. Appl. Meteo., 21, 1594-1609. 